\title{
CHAPTER 5: PHYSICS OF ENERGETIC IONS
}

\section{$\underline{5.1 \text { Introduction }}$}

The development of a basic understanding of the behaviour of burning plasmas, i.e. plasmas with strong self-heating, represents the primary scientific challenge faced by ITER and fusion research in general, and a necessary step towards the demonstration of fusion as a source of energy. In D-T plasmas, such as foreseen for ITER, self-heating is provided by the alphas generated at $3.5 \mathrm{MeV}$ by the $\mathrm{D}-\mathrm{T}$ fusion reactions. Furthermore, other fast or energetic ions with energies in the $\mathrm{MeV}$ range, well above the thermal distribution of the plasma bulk, are generated by Ion Cyclotron Resonant Heating (ICRH) and Neutral Beam Injection (NBI). These are expected to play a major role in achieving and controlling optimal burning plasma scenarios with external heating and/or current drive. Understanding the behaviour of the alpha particles and of other energetic ions is therefore crucial. In particular, a good confinement of the alphas is essential to achieve a high fusion power gain, an essential element of the ITER project.

Transport and confinement of fusion alphas not only impact machine performance by affecting the fusion yield. Due to the large power carried by the alpha population (of order of $100 \mathrm{MW}$ in medium fusion gain discharges in ITER), even relatively small alpha losses can damage the machine first wall and must be avoided. On the other hand, an excessive confinement in the plasma core of the thermalized alphas (helium ashes) would give rise to a dilution of the fuel and should be prevented. The confinement of alphas may be reduced by a combination of orbit losses due to an imperfect toroidal field (the so-called ripple losses) and collective instabilities. Similar considerations apply to fast ions produced by additional heating systems, which have dynamic properties and physical interactions analogous to those characterizing fusion alphas. However, contrary to fusion alphas, their phase space distribution is strongly anisotropic, with the NBI produced fast ions flowing predominantly parallel to the magnetic field, and the ICRH accelerated ions characterized by large perpendicular energy and mostly trapped orbits.

In addition to the interaction of alpha particles with plasma waves and instabilities, possibly leading to their redistribution and losses, a number of new phenomena are expected in a burning plasma, including the effects of alpha particles on macroscopic plasma stability and their transport induced by background plasma turbulence. A burning plasma is a complex self-organized system, providing a great challenge for both experimental and theoretical plasma physics, as well as a unique opportunity to investigate a vast class of problems, ranging from basic science to applied physics.

This new field of research can only be fully assessed in an integrated way in ITER, in which the burning plasma regime will be reached, with a large energy content of the fast ion population. Nevertheless, significant progress in the understanding of burning plasmas has been made in the past few years by investigating weakly self-heated plasmas and plasmas in which fast ions are produced by additional heating schemes.

This separation of the various building blocks related to fast ion physics phenomena was achieved due to an increased level of fundamental understanding, gained through systematic comparisons between a large range of experimental results and increasingly sophisticated theoretical and numerical modelling, including nonlinear wave-particle interaction physics. 
In this Chapter we review the progress accomplished since the redaction of the first ITER Physics Basis. Extended reference to the literature produced in that period is made within the individual Sections. The structure of the Chapter is conceived to present this recent progress in the clearest possible way, and does not pretend to reflect the overall structure of this large field of research. For areas in which we judge that no significant improvements were obtained in the knowledge or in the control capabilities, for example for the background transport of fast ions or for the possible impact of ion cyclotron emission, we simply refer to the former ITER Physics Basis.

A large and successful effort was dedicated to the development of methods to simulate fusion born alphas in plasmas without significant fusion reactivity. The main parameters determining the amount of free energy available for alphas to drive instabilities, $\beta_{\text {fast }}$ and $R \nabla \beta_{\text {fast }}$, have reached values close to or even exceeding those predicted for ITER. Alfvénic Mach numbers, i.e. particle velocities normalized to the Alfvén speed, are essentially the same as in ITER. On the other hand, two of the parameters important in the fast ion physics are still significantly different from ITER. First, the ratio between the alpha (or fast ion) slowing down time and the energy confinement time, determining the time scale for the response of the plasma temperature to the plasma self-heating regulated by the alphas, is much larger in present devices than in ITER. Second, the normalized (inverse) fast ion Larmor radius, namely the number of fast ion gyro-orbits contained within the plasma radius, is much smaller than foreseen in ITER. Such a parameter is important in determining the range of most unstable mode numbers for alpha driven instabilities, as well as the simultaneous presence of a large number of unstable Eigenmodes. New techniques using trace levels of tritium and simulated (i.e. not produced by fusion reactions) alpha particles have been developed, providing information on the confinement of the alpha particles over a wide range of conditions. The different methods to generate fast ions that are available in present devices are reviewed in Section 5.2.

Ripple losses are relatively well understood, owing to important new experimental results, e.g. from JFT-2M, and to comparisons with numerical codes. The synergy between new observations and advanced modelling has led to an optimization of ferritic inserts in ITER to reduce ripple alpha losses with reversed shear configurations by more than one order of magnitude. The advances in the physics of ripple losses are discussed in Section 5.3.

Section 5.4 deals with the interaction of fast ions with MHD activity and related transport. The interaction of fast ions generated by additional heating methods with low frequency MHD could be investigated in a variety of experiments. Nonlinear modelling appears to be in qualitative agreement with experiments, although more work remains to be done in quantitative predictions. The linear theory of kinetic ballooning modes and localized interchange modes is well advanced, and the behaviour with sawteeth (including 'monsters' and neo-classical tearing modes) is well understood qualitatively. A quantification of the effect of background turbulence on the behaviour of fast ions calls for a dedicated combined theoretical and experimental effort.

The field of linear stability thresholds for collective instabilities (Section 5.5) was substantially advanced through a large number of experimental results and significant progress in theoretical simulations. New experimental techniques to launch and detect stable modes have led to large experimental databases of damping rates and to information on the fast particle drive of modes of low toroidal mode numbers in the Alfvén Eigenmode 
frequency range. Improved fast ion sources (discussed in Section 5.2) and extended high frequency fluctuation measurements have provided large amounts of data on the instability thresholds, for a large range of toroidal mode numbers. Damping and drive mechanisms are qualitatively understood, although quantitative predictions for specific modes are still to be ameliorated, especially in regimes in which fluid and kinetic models give significantly different results. This can be the case when the mode is very close to marginal stability or when the mode structure is spatially extended and wave propagation properties as well as non-local interactions with the shear Alfvén continuous spectrum are important. Separate considerations are needed for modes excited significantly above marginal stability, such as the Energetic Particle Modes, whose properties depend upon the fast particle profile and which can be resonantly driven at the characteristic frequencies of fast ions.

Rapid progress was also achieved in the study of the nonlinear phase of the interaction between waves and fast ions, particularly in the weakly nonlinear regime, as discussed in Section 5.6. The understanding of the linear and nonlinear wave-particle interaction has reached a stage at which measurements of the modes are used to extract information about the background plasma and/or the fast ion population. For example, Alfvén Cascades, i.e. modes with frequency sweeping up to the Alfvén Eigenmode gaps, are observed extensively in discharges with non-monotonic safety factor profiles, and used to get information on the temporal evolution of the safety factor itself. Limited information, on the other hand, is available on the fast ion redistribution and losses, due to the difficulty in achieving large amplitude modes in present devices and in having sufficiently sensitive diagnostic tools to measure the energy and radial distribution of the fast ions. Significant redistribution of fast ions over short time scale could only be inferred indirectly in the case of Energetic Particle Modes, by comparing the measured evolution of the mode spectra with the predictions of numerical simulations. Some information about macroscopic energetic particle losses on short time scales can be obtained experimentally by comparing fast ion radial profiles before and after large bursts of Alfvénic activity, such as in the case of the Abrupt Large amplitude Events observed on JT-60U.

Section 5.7 concludes this Chapter, providing a summary along with concluding remarks and elements for discussions.

\subsection{Sources of Energetic Ions}

There are three main sources of energetic ions in tokamaks: fusion reactions, injected neutrals, and ions accelerated by radio frequency waves in the ion cyclotron (ICRF) and lower hybrid ( $\mathrm{LH})$ range of frequencies. Although these sources were already well understood and extensively tested [1] at the time of writing of the previous ITER Physics Basis [2, 3], the intervening years have seen further demonstrations and applications of each of them.

The largest alpha particle populations in TFTR and JET experiments had already been formed at the time of the last review. Parameters that relate to instabilities driven by fast ions are compiled for these discharges in Table 5.1. More detailed TFTR studies of the tritium beam ions $[4,5]$ and of the alpha particles [6-9] appeared subsequently. Recent JET contributions include new diagnostic techniques related to alpha particles and tritons [10-14].

Intense populations of anisotropic, predominantly perpendicular, fast ions with large effective temperatures have been created by ICRF heating for decades. Both fundamental minority 
heating and heating at harmonics of the cyclotron frequency are effective. Acceleration of beam ions at the third, fourth, and fifth harmonics is now an established technique, having been demonstrated in many recent experiments [15-20]. In particular, a ${ }^{4} \mathrm{He}$ beam was accelerated to $\mathrm{MeV}$ energies in JET [19]. The parameters of the alpha populations produced by these methods are comparable to those that characterize the alpha particles generated during DT operation (Table 5.1). The fast-ion density profile can be modified by varying the ICRH antenna phasing, i.e. the parallel wave number [21-23]. When the energetic tail ions are ${ }^{3} \mathrm{He}$ or ${ }^{4} \mathrm{He}$, gamma ray tomography is a powerful new technique to measure the fast-ion profile [24]. Experiments involving ICRF acceleration of helium could be employed to test alpha particle diagnostics prior to the introduction of tritium in ITER.

Lower hybrid current drive is a candidate for current profile control in ITER [3]. Absorption of the wave energy by alpha particles could have an adverse effect on current drive efficiency [25]. For example, in JET [26], the MeV hydrogen tail produced by fundamental ICRF heating absorbed more wave energy than predicted by cold plasma theory. However, further experiments are needed to quantify the role of fast ions in the Lower hybrid wave absorption process in different plasma scenarios.

Neutral beam heating, including development of negative ion beams (N-NBI), was previously reviewed [3]. Recent studies confirm that the calculations of beam deposition are accurate and reliable [27-28]. Improvements in the parameters of N-NBI heating on JT-60U (in terms of beam energy and current) have allowed for a number of simulation experiments of relevance to ITER [29-33]. In particular, the measured neutral beam current drive efficiency is in good agreement with theoretical predictions for $\sim 350 \mathrm{keV}$ deuterium beams in the absence of strong beam-driven instabilities [32]. Circulating beam ions were also employed to study Alfvén instabilities under ITER-like conditions (Table 5.1) [33].

Another advance in the study of energetic ion physics is the application of neutral beam injection to new facilities. For example, because of the low magnetic field in spherical tokamaks, conventional (positive) neutral beam injection routinely produces super-Alfvénic fast-ion populations; a wide variety of beam-driven instabilities are observed [34-43]. Neutral-beam injection into stellarators drives instabilities that shed new light on our understanding of fast-particle driven instabilities [44-50].

\subsection{Ripple induced losses}

Toroidal field (TF) ripple arising from the discreteness of toroidal coils can cause energetic particle losses, commonly referred to as 'ripple losses'. The resulting heat load on the first wall limits the allowable TF ripple amplitude in a tokamak fusion reactor, thereby setting a lower limit on the number of coils. The major points that were understood on ripple losses at the time of the publication of the ITER Physics Basis were: 1) ripple losses are numerically predictable as indicated by experiments in JET [52], JT-60U [53] and TFTR [9]; 2) ripple losses of $\alpha$-particles in ITER are anticipated to be negligible in discharges with monotonic, positive magnetic shear [2];3) on the other hand, the losses can be a concern in advanced operation scenarios based on reversed shear. Since the writing of the ITER Physics Basis, publications on ripple loss experiments have appeared for TFTR [5,9,54-55], JFT-2M [56-58] and Tore Supra [59-60].

An important issue in recent research is the effect of the safety factor (q) on ripple-induced 
transport. For high q values, the magnetic perturbation due to TF ripple along the field line increases, leading to an enhanced ripple transport. Furthermore, high q values lead to an expansion of the ripple-trapping region and the Goldston-White-Boozer boundary for stochastic ripple diffusion [61]. Enhanced ripple losses in reversed shear plasma were indeed observed experimentally [5,9,54-55,62-63]. The observed enhanced losses of energetic alpha particles and tritons in reversed shear are basically explained by the existing codes $[9,54,62]$. In the TFTR beam blip experiment in reversed shear, however, the observed loss of neutral beam injected ions was about a factor of two larger than expected [5]. A possible clue to this anomaly is the anisotropy of the particle source in the velocity space, but the issue has not been fully resolved to date. As enhanced alpha particle losses can constitute a critical problem in advanced operation, significant effort has been dedicated to reducing the amount of TF ripple in ITER.

\subsubsection{Ripple reduction with ferritic steel inserts}

When ferritic steel (FS) plates are placed between the plasma and each TF coil, the magnetic field amplitude below each TF coil is reduced. Therefore FS plates with appropriate thickness can be used to flatten the magnetic field strength and minimize the magnitude of the TF ripple. FS inserts were used successfully to reduce ripple losses in the JFT-2M experiment, although the TF ripple structure was not fully optimized, due to space limitations imposed by the existing vessel ports. The resulting complex TF ripple structure generates two concerns. First, enhanced transport can result from higher harmonics of the ripple, as quantified by the predicted scaling of the diffusion coefficient of energetic particles with the toroidal harmonic number $\mathrm{N}$ and the amplitude of TF ripple, $\delta: \mathrm{D} \propto \mathrm{N}^{9 / 4} \delta^{3 / 2}$ [64]. In practice, for ITER, this effect is not anticipated to be quantitatively significant. Second, a non-periodic, highly localized TF ripple could lead to additional losses [58]. This kind of localized ripple can be produced around a large tangential port where an irregular arrangement of FS plates is required because of space constraints. Figure 5.1(a) and (b) show the arrangement of FS inserts in JFT-2M, the resulting TF structure and localized ripple well in experiments dedicated to investigating this effect. The measured energetic particle losses by ripple trapping are shown in Fig. 5.1(c). The ripple-trapped losses are roughly proportional to the thickness of the local ripple well. The experimental results were well reconstructed using a full three-dimensional, orbit-following, Monte Carlo code (F3D-OFMC), which treats the three-dimensional TF ripple distribution and the wall structure. It is confirmed that the FS inserts in ITER do not produce such a highly localised ripple.

\subsubsection{Code development}

The existing Monte Carlo codes for ripple loss calculation (OFMC [65] and HYBRID [66]) have been modified to treat the complex rippled field produced by FS plates. In both codes, the complex rippled field is obtained by a nonlinear magnetic field calculation, prior to the orbit calculation. In the case of OFMC, an optimal arrangement of FS plates is determined to minimize the average TF ripple on a given surface in the plasma, referred to as the evaluation surface. Usually the evaluation surface is placed on the plasma surface. Starting from the allowable installation spaces, the board size and maximum thickness of the FS boards, the optimal arrangement of FS inserts is determined by a three-dimensional nonlinear magnetic field analysis [67-68]. Once the optimal arrangement of FS inserts is determined, the resulting magnetic field is Fourier decomposed, to perform the calculation of the ripple losses. A similar approach is taken in the HYBRID code.

The two codes provide similar estimates of the $\alpha$-particle losses in ITER positive shear and reversed shear plasmas [68]. The OFMC code has recently been upgraded to treat a three- 
dimensional TF ripple distribution and a realistic wall structure (F3D-OFMC) [58]. Nevertheless, the use of the code for calculating the beam ion losses in present tokamak experiments has been so far very limited.

\subsubsection{Projections to ITER}

The ITER vacuum vessel has a double wall structure, with the gap between the inner and outer shells filled with steel and water for neutron shielding. Present plans are to install FS (SS430) plates in this shielding located between the vessel double shells. If the assembly of the in-wall steel is used with an appropriate combination of ferritic and austenitic steel plates, the TF ripple can be reduced without affecting the properties of the shielding itself. Naturally, the vessel ports impose a geometrical constraint for the arrangement of the steel plates. Figure 5.2 shows a comparison of the TF ripple distribution in ITER with and without FS inserts, indicating that TF ripple is drastically reduced with FS inserts [67]. Here, the FS region is divided into eight portions with a uniform filling factor of 0.23-0.61 in each portion.

The HYBRID simulations indicate that $\alpha$-particle (power) losses will be $9.3 \%$ in strong reversed shear of ITER for relatively peaked $\alpha$-source without ferritic inserts, and that these losses can be reduced to $0.13 \%$ if FS inserts are installed. As a consequence, the resulting heat flux can be reduced from 0.8 to $0.025 \mathrm{MW} / \mathrm{m}^{2}$, a value that is much smaller than the allowable heat load of the first wall $\left(\sim 0.1 \mathrm{MW} / \mathrm{m}^{2}\right)$. The OFMC calculation for a similar ripple distribution shaped with a different FS arrangement also indicates a reduction of $\alpha$-particle losses by more than one order of magnitude with FS inserts, and that the power loss fraction is less than $1 \%$ even for a uniform $\alpha$-source in reversed shear plasmas with $\mathrm{q}_{\min } \leq 3$ [68]. The heat load due to $\alpha$-particle ripple loss predicted by the OFMC code is depicted in Fig.5.3, indicating consistency with the HYBRID simulation.

\section{$\underline{5.4 \text { Fast ion interaction with MHD and transport }}$}

Additional heating and fusion reactions can generate large amounts of fast ions in the core of the tokamak plasma discharge. These ions can affect the MHD stability of the discharge and, in turn, can be affected by MHD instabilities during their slowing down. It is important to understand and possibly control the transport of the fast particles, as it determines the efficiency with which those particles heat and drive current [32]. Manipulating the fast particle spatial and energy distributions can in principle lead to controlling the plasma burn or even to channelling energy from the fast particles to the fuel ions using waves [69].

These effects are discussed in this Section for the low frequency part of the MHD spectrum, while resonant wave-particle processes, leading to the excitation of MHD instabilities and to fast particle redistribution and losses, which generally characterize higher frequency MHD, are discussed in the following Sections.

Sawtooth oscillations are the result of internal reconnection events that locally break the magnetic topology and cause a sudden loss of confinement. This core-localised loss may not affect the overall performance significantly, but sawteeth can affect ITER performance by coupling to other non-ideal MHD instabilities, such as Neoclassical Tearing Modes (NTMs). In addition, sawteeth strongly interact with fast particles, such as ions energised by ICRH or fusion produced $\alpha$ 's, in two ways: they can be stabilised by them and they can affect their radial profile. 
At the time of the previous ITER Physics Basis [2], the advantage of sawtooth suppression, leading to more peaked profiles and enhanced ignition margins, was weighted against the negative effects of larger sawteeth crashes. Although it was expected that ignition would be maintained despite the redistribution of alpha heating power, the enhanced heat load to the wall or even a disruption resulting from the triggering of NTMs, big ELMs or other MHD events by the big crash was a concern. The control of the stability of the sawteeth through auxiliary heating was briefly addressed, as well as the possibility to make discharges with $\mathrm{q}_{0}>1$, avoiding sawteeth altogether. Substantial progress has been made in these areas, owing to an increased capability of producing and diagnosing fast ions in the relevant energy range (see Section 5.2), both by additional heating and by fusion reactions, and to advances in the theoretical modelling.

The stabilization of sawteeth through fast particles has been studied further recently with NBI ions on DIII-D [16] and JET [70] and with negative N-NBI beams on JT-60 [71]. Alpha particles in JET also provide a significant stabilizing contribution. Record fusion yields were obtained on JET in discharges where the sawtooth was delayed [72].

Theoretical progress was made towards an understanding of the evolution of the sawteeth, their stabilization and the mechanism of the crash [73,16,70]. Energetic Particle Modes (EPMs) were proposed as the leading cause of the monster sawtooth crash [74-75]. A sawtooth model accounting in a simplified way for the redistribution of fast ions at the crashes was developed [15], and the poloidal distribution of the alphas to the wall was calculated for TFTR discharges [76]. More detailed models evaluate the fast particle redistribution for different values of energy and of the ratio of toroidal drift velocity to longitudinal velocity [77]. The role of the electric field induced by the reconnection has been highlighted [78].

Experimental results on the redistribution of fast particles due to the sawteeth include data on alpha particles [8] and deeply trapped $\mathrm{RF}$ driven $\mathrm{H}^{+}$ions. The latter are redistributed well beyond the $\mathrm{q}=1$ surface [79], although there is a strong indication that a substantial fast particle component can remain in the centre [80]. This is in agreement with a model predicting that trapped particles with sufficiently high energy, hence high precession frequency, are not sensitive to sawtooth crashes [81].

In summary, although certain fundamental issues relating to the sawtooth crash remain unresolved, progress in understanding some aspects of the MHD instability that leads to sawtooth crashes has been sufficient to allow scenarios for controlling the sawtooth period to be designed theoretically and implemented in experiments. The redistribution of fast particles following sawtooth crashes is qualitatively understood. However, as fast particles strongly stabilise sawteeth, long sawtooth periods are expected in burning plasmas, leading to large crashes that can trigger NTMs [82] at relatively low $\beta$ values, unless measures are taken to control sawtooth activity.

Due to the importance of NTMs, whose growth can lead to operational limits in ITER, the effect of the sawtooth crash on triggering NTM [83], reducing the overall confinement and even the driven current [84], has become a concern. A number of methods can be used to avoid the detrimental effects of a large sawtooth crash. The amplitude of sawteeth can be controlled using ICRF [85]. In this case, the onset of NTM can be delayed or avoided [86] leading to higher beta values [87]. Small sawteeth can appear spontaneously at low density, when the fast-ion energy contribution to the total plasma diamagnetic energy content exceeds 
$45 \%$ [88]. Sawteeth can also be avoided altogether by maintaining $\mathrm{q}_{0}>1$ [89-91], although the corresponding low shear does not necessarily imply the absence of MHD activity [92-93, 45].

Large fast particle pressures in the plasma core can directly lead to low frequency MHD instabilities, such as the fishbones [94-95, 41, 47]. Fishbones are observed to trigger sawteeth [96] and, similarly to sawteeth, can be associated with magnetic reconnection [97], eject different types of fast ions [47], trigger NTM [98] and substantially reduce the confinement and the neutron rate [99].

Recent theoretical work [100] has also indicated that the kinetic effects associated with the alpha and thermal particle species can significantly affect the stability of the Resistive Wall Mode in a burning plasma. Such a stabilizing effect is induced by the precession motion of the trapped particles (of both the alpha and the thermal species), and becomes important for plasmas (such as ITER's) rotating with a relatively low frequency below the ion diamagnetic frequency.

The fast particle transport can be controlled indirectly by influencing the MHD activity, as discussed above, or by acting on the different additional heating methods [101-102]. For example, ICRF waves with a momentum directed in the co-current direction will lead to an inward pinch of the particles [103-104], which can be used advantageously to improve the formation of the transport barrier in reversed shear discharges [22].

Finally, a quantification of the effect of background (drift wave like) turbulence on the behaviour of fast ions, as well as the effect of the fast ions on the turbulence, remains for the moment beyond reach for the existing experiments and calls for a dedicated combined theoretical and experimental effort. Although early observations indicated that the energetic particle diffusion coefficient should not be strongly affected by drift waves [1], such interaction may play a role in burning plasma regimes, for example providing a tight connection between the transport of fast particles, on one hand, the plasma rotation and the external current drive efficiency, on the other [105-106].

\subsection{Linear stability of fast particle driven collective modes}

The goal of linear theory of collective modes, in conjunction with ad hoc experimental measurements, is to describe their dispersion properties and to identify and quantify the main mechanisms that damp or drive instabilities in the presence of energetic ions. This physics understanding can be used to predict tokamak operating regimes where collective modes are stable or only weakly unstable and do not alter the alpha particle confinement enough to affect the energy transfer to the bulk plasma.

The presence of energetic ions in tokamak plasmas can result in various instabilities ranging in frequency from close to zero to harmonics of the ion cyclotron frequency [107]. Typically, instabilities in the Alfvén frequency range are driven by the fast ion pressure gradient and may lead to the spatial redistribution or losses of fast ions. Instabilities with higher frequencies, of the order of (or above) the ion cyclotron frequency, are generally driven by velocity space gradients. In both cases, these instabilities provide useful information to diagnose the distribution of the confined alpha particles. Since the publication of the ITER Physics Basis, research has focused on the Alfvén frequency range $\left(\omega \cong \mathrm{v}_{\mathrm{A}} / \mathrm{qR}\right)$, where different types of Alfvén Eigenmodes (AEs) can interact resonantly with particles travelling at 
the Alfvén speed. This is because present day experiments show that the Alfvén type instabilities, such as Toroidal AEs (TAEs), are the most efficient in transporting the energetic ions [108]. To become unstable, the global drive for such modes has to exceed the global damping from the background thermal plasma and the fast ions themselves.

The drive from energetic ions is proportional to their pressure gradient and depends on the fast ion orbit width:

$$
\gamma=\left(\omega_{*}-\omega\right) \mathrm{F}\left(\mathrm{v}_{\text {fast }} / \mathrm{v}_{\text {Alfven }}\right) \mathrm{G}\left(\Delta_{\mathrm{b}} / \Delta_{\mathrm{m}}\right)
$$

where $\omega_{*}$ is the fast ion diamagnetic drift frequency and $\mathrm{v}_{\text {fast }}$ the magnitude of the fast ion velocity. The function $\mathrm{F}$ depends on the exact shape of the fast ion distribution function and the function $G$ on the value of the orbit width parameter $\left(\Delta_{b} \cong q \rho_{\text {fast }}\right.$ for passing particles) relative to the radial scale of the mode with poloidal harmonic number $m\left(\Delta_{\mathrm{m}}\right) . \mathrm{G}=1$ if $\Delta_{\mathrm{b}}<(\mathrm{r} / \mathrm{R}) \Delta_{\mathrm{m}}$ and $\mathrm{G}=\mathrm{O}\left(\mathrm{m}^{-1}\right)$ if $(\mathrm{r} / \mathrm{R}) \Delta_{\mathrm{m}}<\Delta_{\mathrm{b}}<\Delta_{\mathrm{m}}$. This latter 'plateau' regime gives the most unstable toroidal mode number and determines the scaling of the drive as a function of the device size through the relation $k_{\perp} \rho_{\text {fast }} \approx 1$ ( $\rho_{\text {fast }}$ is the fast ion Larmor radius) [109]. Finally, $\mathrm{G}=\mathrm{O}\left(\mathrm{m}^{-2}\right)$ if $\Delta_{\mathrm{b}}>\Delta_{\mathrm{m}}$ [110]. Naturally, the first condition is that the drive should exceed the damping from the fast ions themselves, i.e. $\omega<\omega_{*}$. For the background thermal ions $\omega>>\omega_{*}$ and $F \sim \exp \left(-1 / 9 \beta_{\mathrm{i}}\right)$ for ITER conditions, while $\mathrm{G}=1\left(\beta_{\mathrm{i}}\right.$ is the average ion $\left.\beta\right)$. This results in ion Landau damping, which is the main damping mechanism for low frequency AEs in burning plasmas with relatively large $\beta_{i}$ [111]. For higher frequencies and smaller wavelengths, the electron Landau damping becomes important due to the effect of the parallel electric field [112]

$$
\gamma / \omega \sim\left(\mathrm{k}_{\perp} \rho_{\mathrm{s}}\right)^{2} \exp \left(-\left[\mathrm{v}_{\text {Alfven }} / \mathrm{v}_{\text {electron }}\right]^{2}\right)
$$

where $\rho_{\mathrm{s}}$ is the ion sound Larmor radius. According to PENN gyro-kinetic model [113], the small radial wave field structures of AEs correspond to relatively large perpendicular wave vectors $\mathrm{k}_{\perp}$ and, via Faraday's law $\left(\mathbf{k}_{\perp} \times \mathbf{B} \sim \mathbf{E}_{/ /}\right)$, to a finite perturbed parallel electric field. This may explain why the electron Landau damping can be orders of magnitude smaller when using a fluid rather than a gyro-kinetic description of the wave field and is likely to be the main source of discrepancy between different codes at present. In fluid models the effect or the parallel electric field is introduced either by extending MHD equations to include first order FLR correction [114] or perturbatively, as in NOVA, by applying analytical theory in which $\mathbf{E}_{/ /}$induced radiative damping as well as $\mathbf{E}_{/ /}$contribution to electron collisional [115] and Landau dampings account for additional dissipation [116]. This contributes to the stabilisation of AEs at high-n. Fluid models predict a strong damping from the continuum due to small-scale structures near the resonances. It turns out that in burning plasmas trapped electron collisional damping is dominant even for moderate n's (5 to 10) at the plasma edge [111].

Gyro-kinetic PENN model accounts for different mode-conversion mechanisms to the kineticAlfvén wave (KAW) [117] and the drift-wave [118]. Some are the kinetic counterpart of fluid phenomena (such as continuum and radiative damping [119-121]). Others are intrinsically different and occur where the spatial scales of the fluid wave field and of the drift-kinetic Alfvén wave coincide [117-118]. The resonant interaction of short spatial scales with the electrons and the ions can result in a large damping (and drive). 
Even though fluid and gyro-kinetic models often agree as far as the Eigenmode frequency, the continuum damping calculated by the gyro-kinetic models can be an order of magnitude larger than the Landau damping of the KAW [122]. It is not a priori possible to say which prediction is more accurate without extensive benchmarks and experimental validation. In addition, the global nature of AEs results in a delicate balance, where the drive provided locally has to be weighted against the damping from different locations. Since the last ITER Physics Basis, a considerable effort has been devoted to trying to disentangle the physical dependences from theory and experiments in order to suggest possible actuators to control the instabilities.

\subsubsection{Identification of Alfvénic modes in different operating scenarios}

The previous ITER Physics Basis describes a variety of Alfvén Eigenmodes that are associated with the plasma current and density profile (GAEs), the shape (TAEs, EAEs) and kinetic effects (KAEs, KTAEs) in conventional positive shear scenarios. New modes have since been discovered, mainly in advanced scenarios, using auxiliary power to tailor the current profile and raise the plasma beta.

Non-perturbative studies of the resonant character of beta-induced Alfvén Eigenmodes (BAEs) in DIII-D have been performed in an attempt to identify modes that are driven by beam ions and the frequency of which seems to coincide with the Alfvén continuum. In weakly reversed shear discharges, TAEs and BAEs have been observed with the BAE frequency $(\sim 100 \mathrm{kHz})$ about half of TAE frequency $(\sim 200 \mathrm{kHz})$. Earlier numerical computations, however, failed to establish conclusively the identity of this instability [123]. A theoretical analysis has been performed with HINST [124], a fully kinetic code that can reproduce both the resonant TAEs and other AE branches. The major limitation of the code is that it solves for a radially localized solution in ballooning coordinates and cannot compute the full two-dimensional eigenfunction for conditions of low shear and intermediate mode numbers $(n=3-5)$. The analysis suggests that two types of energetic particle (or resonant) modes are observed in DIII-D plasmas with frequencies inside the lower Alfvén continuum: the resonant TAE (a specific kind of Energetic Particle Mode in the TAE frequency range, see Section 5.6.4) and the resonant kinetic ballooning mode (r-KBM). The drive from beam ions is sufficient to overcome the continuum damping. The r-KBM is transformed into a KBM, which is stable in the absence of fast beam ions, seems to be responsible for the experimentally measured modes (generally dubbed as BAEs). The KBM frequency depends sensitively on the beam-ion distribution function, which differs greatly in different discharges. The beam-ion distribution function almost certainly changes between the measured bursts, which can explain the observed sudden changes in the mode frequency [125-126]. Similar considerations could be made on the basis of Refs. [127-128], which suggest that an accurate modelling of these modes should include the beam ion drive, the thermal ion compressibility and the wave-particle interaction.

The drift-wave character of AEs in deeply reversed shear plasmas with a significant value of the ratio $\omega *_{\mathrm{i}} / \omega_{\mathrm{TAE}} \propto \mathrm{nq}^{2}(\rho / \mathrm{a})^{2}$ has been studied using the global gyro-kinetic PENN code [113]. The computations suggest that drift-kinetic AEs (DKAE) with low toroidal mode numbers can become unstable in a deeply reversed shear ITER plasma with $\mathrm{q}_{\min }>3$ [118]. Low-n DKAEs do not follow a simple scaling with the Alfvén frequency and are not stabilized by the edge magnetic shear in the presence of a magnetic X-point. Experiments conducted in JET confirm that unstable low $n=3-5$ Alfvénic modes can exist without ICRF driven $\mathrm{MeV}$ energy ions and are driven only by NBI with a low beam to Alfvén velocity ratio $\mathrm{V}_{/ / \mathrm{NBI}} \cong 0.3 \mathrm{v}_{\mathrm{A}}$. These modes do not follow the scaling expected for Alfvén waves, appear in a 
diverted plasma and could be unstable DKAEs [129]. A complete identification of the measured stable and unstable modes, however, is still missing.

The original ITER Physics Basis describes a variety of Alfvén Eigenmodes that are mostly associated with magnetic measurements on the exterior of the plasma. Since then, improvements in the diagnostic of fluctuations in the plasma core in large tokamaks have led to the realization that many more modes exist in the plasma core than can be observed at the edge, highlighting the need for core density fluctuation diagnostics on a future burning plasma experiment. These observations also demonstrated that reverse shear plasmas exhibit extensive mode activity in the core with toroidal mode numbers typically exceeding the range theoretically predicted for present day devices.

For moderately reversed shear plasmas, fast particle driven instabilities appear, whose frequency varies in time along with the diffusion of the plasma current. In JET, $n=1-7$ ICRF driven Alfvénic modes originate in the Alfvén continuum around $r / a=0.2$ at $\mathrm{f} \cong 20-50 \mathrm{kHz}$, chirp up at a rate proportional to the toroidal mode number, $\mathrm{df} / \mathrm{dt} \sim \mathrm{n}$, and eventually merge into a TAE at $\mathrm{f} \cong \mathrm{f}_{\text {TAE }}\left(\mathrm{q}=\mathrm{q}_{\text {MIN }}\right)$ around $\mathrm{r} / \mathrm{a}=\mathrm{r} / \mathrm{a}\left(\mathrm{q}_{\mathrm{MIN}}\right) \cong 0.5$. Dubbed Alfvén cascades, such modes have been modelled as global modes that closely follow the edge of the continuum [130-131], or DKAEs in deeply reversed shear configurations [118]. While under most common JET conditions Alfvén cascades are characterized by upward frequency sweeping, these modes may be characterized by both an up- or downward sweeping in frequency that depends on the details of the equilibrium and fast ion distribution function [132-133]. In addition, theory predicts that a strong and localized fast ion drive may resonantly excite EPMs with weak continuum damping (low threshold) in the plasma core [134].

In JT-60U, two frequency-sweeping modes sometimes appear simultaneously during negative-ion based neutral beam injection (N-NBI) and merge into a TAE. Figure 5.4 illustrates how an $n=1$ instability sweeps up through the range $40-90 \mathrm{kHz}$ while another sweeps down through 130-90 kHz over the period from 6-6.5 sec, when both merge into a single mode [135]; the mode amplitude is enhanced when the frequency chirping saturates. The kinetic full-wave code TASK/WM has been used to interpret the measurements as reversed shear induced Alfvén Eigenmodes (RSAE, an alternative name for Alfvén cascades) located immediately below the $\mathrm{m}=2$ and above the $\mathrm{m}=3$ Alfvén continua merging into a $(3,2)$ TAE where $\mathrm{q}_{\mathrm{MIN}} \cong 2.5$ [136]. Figure 5.5 shows in a sketch how two RSAEs sweep in frequency $\mathrm{f}_{\mathrm{RSAE}} \cong\left(\mathrm{n}-\mathrm{m} / \mathrm{q}_{\min }\right) \mathrm{v}_{\mathrm{A}} /(2 \pi \mathrm{R})$ and $\left.\mathrm{f}_{\mathrm{RSAE}} \cong\left((\mathrm{m}+1) / \mathrm{q}_{\min }-\mathrm{n}\right) \mathrm{v}_{\mathrm{A}} / 82 \pi \mathrm{R}\right)$ as the value of $\mathrm{q}_{\min }$ drops and the gap is closing to form a TAE at $f_{T A E} \cong v_{A} /\left(4 \pi q_{T A E} R\right)$, where $q_{T A E}=(m-1 / 2) / n$.

These findings led to a re-interpretation of TFTR-DT data using reflectometry to reconstruct the internal structure of alpha particle driven modes [137]. These modes were originally considered to be TAEs [138], with a caveat associated with the anti-ballooning structure of a low frequency $n=2$ mode [139]. The observation of an anti-ballooning density mode structure for the low frequency $n=2$ mode has been highly controversial for some time but was resolved when the NOVA-K code suggested that the modes could be RSAEs, which near $\mathrm{q}_{\min }$ should exhibit density peaking on the high field side of the magnetic axis (Fig. 5.6).

In recent JET experiments, a broad spectrum of cascade modes was observed in reverse magnetic shear plasmas by operating the plasma density below the O-mode reflectometer cutoff density (Fig. 5.7) [140]. The interferometer data revealed a rich Alfvén spectrum consisting of many frequency-sweeping discrete modes ranging from $40 \mathrm{kHz}$ to the TAE frequency $\approx 140 \mathrm{kHz}$. These observations are in agreement with the established characteristics 
of Cascade or RSAE modes driven by high-energy hydrogen minority ions heated by the fast waves. The new evidence from the plasma core indicates that Cascades are far more prevalent in advanced tokamak regimes and in higher numbers than previously thought, with mode numbers up to $n=16$ observed in the plasma core. In a parallel development, the phase contrast imaging (PCI) diagnostic on Alcator C-Mod has been successful in identifying mode activity not observable on external magnetic probes [141]. Both in JET and in Alcator C-Mod, core chirping modes are observed that cannot be identified on external magnetic probes. A recent analysis on the DIII-D device has shown similar RSAE activity excited by beam ions travelling close to $1 / 3$ of the Alfvén velocity [142]. The excitation of many high-n modes is occasionally observed on the DIII-D facility, however an understanding of the relevant dominant drive and damping mechanisms is still to be reached. An investigation of these modes by multiple radial and vertical interferometer measurements on DIII-D has highlighted the need for a detailed study of the optimum interferometer arrangement for measuring internal modes in ITER [143].

\subsubsection{Alfvén Eigenmode damping and drive mechanisms}

While the frequency is often sufficient to identify an Eigenmode, it is the balance between the damping rate and the fast particle drive that determines the mode stability. To investigate the physics of the mode stability directly one needs experimental information on the damping and drive independently, even in the absence of large populations of resonant fast particles, which will not be available before actual burning plasma experiments such as ITER. This motivated the development on JET of a dedicated active diagnostic system for the excitation and detection of MHD modes in the Alfvén frequency range [144-145]. Considerable progress in the understanding of the dominant mechanisms has been made by comparing active measurements of global damping rates of low-n modes in JET with calculations from global wave field codes such as Castor-K [119], PENN [113] and NOVA-K [146], although more work remains to be done on the benchmarking of the different codes with each other and with analytical theory.

In conventional scenarios, high magnetic shear in the plasma periphery inducing mode conversion to the kinetic Alfvén wave has been identified as the main factor causing strong damping for radially extended AEs from low $n=1$ [117] to intermediate mode numbers $n=12$ [118]. This occurs even in the absence of shear Alfvén resonances and can in principle be controlled externally by varying the plasma shape. Figure 5.8 illustrates this mechanism for an $n=6$ AE in JET, where the wave field extends radially to the magnetic X-point and couples to the kinetic Alfvén wave visible in the divertor region at the bottom of the plasma. Even if the $\mathrm{AE}$ is locally driven by the pressure gradient of fast ions in the plasma core, the strong electron Landau damping of the kinetic Alfvén wave in the edge region can overcome the drive and stabilize the mode. The phenomenon has been studied in detail on JET for the $n=1$ TAE and the $n=0$ GAE by varying the plasma shape at almost fixed $q 95 \cong 4, q 0 \cong 0.9$ and suggests the possibility of controlling the stability of AEs in real time [147]. Figure 5.9 shows the dependence of the measured damping rate separately on the elongation $\kappa$ (averaged over $0.08<\delta<0.12$ ) and triangularity $\delta$ (averaged over $1.1<\kappa_{0}<1.2$ and $1.2<\kappa_{95}<1.3$ ). Notice that when averaging over low values of the plasma elongation, the global damping rate increases approximately linearly with the triangularity and the edge magnetic shear. Conversely, when averaging over low values of the plasma triangularity, the AE damping rate shows a sharp increase for a small variation in the elongation around $\kappa_{95} \cong 1.5$ and $\kappa_{0} \cong 1.35$. These measurements are consistent with the fact that low $n=0-2$ AEs (which tend to be radially extended) have never been observed unstable in conventional positive shear JET plasmas. Moreover, for similar background plasma conditions, 30\% less NBI power and parameters 
further from the resonance condition $\left(\mathrm{v}_{|| N B I} \cong 0.8 \mathrm{v}_{A}\right.$ compared to $\left.\mathrm{v}_{|| N B I} \cong 0.95 \mathrm{v}_{\mathrm{A}}\right)$ are sufficient to destabilize TAEs with intermediate n's in plasmas with low edge magnetic shear, compared with plasmas with high edge magnetic shear [148].

The parametric dependences of this edge damping mechanism have been reproduced within $30 \%$ using the PENN code. According to the PENN code, weak magnetic shear near the axis triggers a coupling to the kinetic Alfvén wave and results in the subsequent electron Landau damping that accounts for most of the global damping of the low $n=1$ AEs, as measured in JET when the stronger mechanism in the plasma periphery is basically absent [149]. Figure 5.10 shows a comparison between measurements and theory for Ohmic limiter discharges with a mix of $\mathrm{H}, \mathrm{D}$ and $\mathrm{T}$ isotopes. Gyro-kinetic calculations reproduce the measurements within $30 \%$ using the mode conversion mechanism in the plasma core, while fluid calculations, which do not include this mechanism, predict damping rates that are too small by an order of magnitude. Radiative damping computed using the NOVA-K and PENN codes is typically more than an order of magnitude smaller and does not reproduce the dependences observed for the damping of $n=1$ modes in JET [150]. Contrary to the radiative model assumptions, only a weak dependence of the global damping rate on the normalized Larmor radius has been observed experimentally in ohmic limiter plasmas (Fig. 5.11).

The damping of low-n, low-m TAEs in JET limiter plasmas has been investigated independently with the use of CASTOR-K [119], a code based on reduced kinetic model [120], and a more recent linear gyro-kinetic code LIGKA [121]. In the plasma core, these three codes contain the same key physics as PENN, i.e. the mode conversion mechanism to the KAW. However, the TAE to the KAW conversion in the centre of the plasma, arising from PENN, could not be confirmed by any of these codes or by analytical studies. The calculated damping rates by the reduced kinetic model and LIGKA are about a factor of 8 smaller than the PENN results. It was reported in [149] that with artificial suppression of the core- KAW-mode in PENN the damping rate drops by one order of magnitude. It remains to be resolved whether the nature of this discrepancy is of physical and/or numerical origin.

With an open TAE gap, the antenna version of CASTOR-K found significant damping due to mode conversion at the plasma edge [119]. PENN calculations also report this mechanism but the overall damping is still strongly affected by the core [149]. LIGKA and the reduced kinetic model did not find any significant mode conversion at the plasma edge. This could be attributed to the fact that they are not as complete as the antenna codes PENN or CASTOR-K in the edge region. However, in the case of a closed gap, continuum damping at the edge was found to be the dominant damping mechanism by LIGKA and the reduced kinetic model code, resulting in damping rates up to $1 \%$. When comparing with measured data it should be kept in mind that open and closed gap cases often cannot be distinguished with high confidence because of experimental uncertainties in edge density data at JET.

Increasing the bulk plasma beta causes the fluid AE wave field to peak and, in theory, leads to increasingly global modes with intermediate mode numbers when the kinetic Alfvén wave extends radially to couple adjacent gaps. Such modes represent a serious threat for collective transport, but can be stabilized if they reach a region with larger damping, for example when the wave field extends to the high shear region at the plasma periphery. This happens when the pressure in a conventional single null plasma is increased while keeping the fast particle pressure constant, and it is the reason why intermediate $n=6-12$ AEs get stabilized. The predicted threshold in the normalized bulk plasma beta, $\beta_{\mathrm{N}}$, is in good agreement with the experiment [118]. 
Experimentally, the situation appears more complicated. The damping of the externally driven $\mathrm{n}=1$ TAE on JET, measured in NBI-heated limiter discharges, increases with $\beta_{\mathrm{N}}$ only above a certain value, $\beta_{\mathrm{N}}>0.45$; a splitting of individual modes as $\beta_{\mathrm{N}}$ is increased is also observed [151]. These measurements challenge the predictions from fluid models of an increase in the damping rate with $\beta_{\mathrm{N}}$, but could be qualitatively consistent with the predictions from gyro kinetic models of kinetic AEs (KAE) in plasmas with low edge magnetic shear [152]. On the other hand, the prediction by both fluid and gyro kinetic models that the mode frequency decreases for increasing $\beta_{\mathrm{N}}$ is clearly confirmed experimentally.

Similarity experiments have been performed in NSTX and DIII-D with a reduced magnetic field $\mathrm{B} \cong 0.6 \mathrm{~T}$, using conventional positive shear plasmas to verify the theoretical predictions that the most unstable range of toroidal mode numbers scales with the plasma minor radius [153]. Similar plasma parameters were established with the exception of the major radius and the safety factor. In NSTX, TAEs were observed with $n=1-2$, whereas in DIII-D with $n=2-7$. The data shows equally good correlation with models for the most unstable mode number, proportional to $\mathrm{a} / \mathrm{q}$ and $\mathrm{a} / \mathrm{q}^{2}$. Figure 5.12 displays the most unstable mode number using the dependence $n \cong 1.6 \mathrm{a} \Omega_{\mathrm{c}} / \mathrm{q}^{2} \mathrm{v}_{\mathrm{A}}$. These measurements were further analyzed with the help of the kinetic code NOVA-K [42]. The calculations predict unstable modes in the interval $n=1-3$ for NSTX and $n=4-7$ for DIII-D, although the model underestimates the damping at the low end of the unstable mode range, since $n=2-3$ TAEs are predicted to be unstable even though they were observed to be stable in the experiments. For the medium- to high-n mode numbers, the stability threshold is accurately reproduced, though without the damping mechanisms that are found to be dominant in JET. High-n modes are stabilized by finite orbit width and finite Larmor radius effects. The main damping here comes from the ion Landau and the radiative damping.

Considerable progress has been made for conventional positive shear plasmas, where AEs are generally more stable. More studies are required to extend the AE stability predictions to reversed shear plasmas. From the experiments in JET, JT-60U and DIII-D, it appears that a small fast particle pressure is sufficient to destabilize Alfvénic instabilities with low mode numbers $[125,130,135]$ even in the presence of a magnetic X-point. Unstable Alfvénic modes have also been observed in deeply reversed shear discharges in the presence of ions with velocities of the order of a third of the Alfvén speed [129]. Further comparisons between models and experiments are needed to fully characterize the mode stability properties in reversed shear discharges.

\subsubsection{Extrapolations to ITER in conventional and advanced scenarios}

Based on the comparison between first-principle theoretical models and present day experiments, the mechanisms that affect the stability of Alfvénic modes can be extrapolated to predict the stability thresholds under reactor conditions, although definitive quantitative conclusions are prevented by the remaining discrepancies between the different codes. This can be done using the codes NOVA-K, HINST and PENN, introduced above. It is found that AEs are generally more stable in conventional positive shear plasmas. ITER operation points have been identified, where a substantial alpha particle pressure $\beta_{\alpha}<1 \%$ (at $\operatorname{Ti}(0)=20 \mathrm{keV}$ ) should only weakly excite AEs with few intermediate mode numbers $n \cong 10-12$. In addition, as these modes are radially localised, extensive fast ion redistribution should not be caused, but a quantitative estimate of their effect on the plasma performance still needs to be performed.

Calculations from NOVA-K displayed in Fig. 5.13 show that high energy NBI ions have an 
additional destabilizing contribution, which could extend the range of unstable modes to $n=8$ 17. Radiative and ion Landau damping are the most important damping mechanisms in the plasma core, whereas trapped electron collisional damping dominates at the edge. This is in reasonable agreement with the HINST code, which tends to underestimate the stability because of its local assumption of the wave field. A different picture emerges from the PENN code, where the converted kinetic Alfvén wave is driven by the alpha particles and Landau damped by the bulk species. These mechanisms can stabilize radially extended mode with intermediate mode numbers up to $\mathrm{n} \cong 12$ and could be used to affect the confinement with external means [150].

Too little is known for a reliable prediction in reversed shear plasmas. Experiments in JET and JT-60U show that the instability threshold is generally lower and calculations from the PENN code suggest that radially extended drift-kinetic Alfvén Eigenmodes with low toroidal mode numbers may be destabilized for large values of safety factor $\mathrm{q}_{\min }>3$, even in the presence of a magnetic X-point, which is normally stabilizing in conventional scenarios.

More definitive conclusions on the predicted stability thresholds for intermediate toroidal mode numbers and in reversed shear scenarios could come from comparisons with experimental data on the damping and drive of modes in a more extended range of toroidal mode numbers. To this aim, a new ad hoc antenna system for the active excitation of MHD modes with intermediate n's has recently been installed on JET [154]. Other experiments are also adopting the same technique, including in the high field, high-density regimes of Alcator C-Mod [155].

\section{$\underline{5.6}$ Collective effects and nonlinear fast particle dynamics}

Non-resonant interaction of fast particles with MHD instabilities, including sawteeth or NTMs was discussed in Section 5.4, while Section 5.5 examines the linear stability of fast particle driven collective modes. Here we deal with the nonlinear interactions of a wide range of collective modes that are driven by the fast particles themselves and can be considered a potentially efficient scattering source for alpha particles and other energetic ions.

Low frequency, low mode-number Magneto-Hydro-Dynamic (MHD) modes affecting the global plasma equilibrium are evidently affecting the energetic particle component as well. Fast ions can destabilize particular classes of these modes, primarily via the precession resonance [156-157], and for sufficiently high power density excite a new type of resonant modes [156], such as the fishbones [158]. Low frequency, low mode-number MHD-like modes are analyzed in Section 5.6.1, whereas energetic particle effects on higher modenumber kinetic MHD modes, such as Kinetic Ballooning Modes (KBMs) [159] and localized interchange modes [160-161] are considered in Section 5.6.2.

In a higher frequency range, collective effects are primarily due to modes of the shear Alfvén branch, as these waves have group velocities directed along magnetic field lines and can resonantly interact with $\mathrm{MeV}$ ions moving with $\mathrm{v}_{\|} \approx \mathrm{v}_{\mathrm{A}}$ [162-163]. For these modes, the fundamental excitation mechanism is provided by transit [164-165] and precession-bounce [166] resonances. As in the case of low frequency MHD-like fluctuations, the wave characteristic behaviour gradually switches from that of Alfvén Eigenmodes to that of resonant modes for increasing energetic particle power density. TAEs constitute the most significant example of the former modes [167], whereas the latter are generally dubbed 
Energetic Particle Modes (EPMs) (or resonant TAEs if they lie in the TAE frequency range, as discussed in Section 5.5.1) [168]. The qualitative differences between AE and EPM linear mode structures and frequency spectra (see Section 5.4) reflect on different nonlinear behaviours. AE nonlinear dynamics is dictated by wave-particle trapping [169-170], as discussed in Section 5.6.3. EPMs, meanwhile, are regulated by their resonant character [171173] and their nonlinear behaviour is strictly related to a redistribution of the energetic ion source [174]. These issues are investigated in Section 5.6.4. Nonlinear mode-mode coupling may also be important, especially in the case of AE, but this topic is not analyzed here. A fairly complete summary of work in this area can be found in [175] and references therein.

The investigation of nonlinear fast particle dynamics in burning plasmas raises the crucial issue of the system behaviour near marginal stability. The possibility of having explosive nonlinear behaviour at moderate growth rates [176-177] may prevent the formation of strongly unstable fast ion distributions, which are required for EPM excitation. On the other hand, the experimental observation of long-lived benign nonlinear $\mathrm{AE}$ structures and of a macroscopic energetic particle redistribution associated with bursting fluctuations [1,178-179] suggests that the strength of the instability depends on the specific fast ion source. Fusion alphas and N-NBI ions, directly generated at $\mathrm{MeV}$ energies, are expected to behave differently from ICRH fast ion tails, which form gradually from thermal energies via resonant wave kicks.

The self consistent analysis of the formation of the energetic particle source, accounting for collective plasma behaviours on the Alfvén time scale and of fast particle Coulomb collisions with the thermal plasma, remains a major open question in burning plasma physics. Meanwhile, detailed analyses of AE dynamics near marginal stability, as well as of EPM nonlinear response in strongly unstable scenarios, can provide useful information on collective effects and nonlinear fast particle dynamics, which may be used as a diagnostic tool, as discussed Section 5.6.5.

\subsubsection{Low frequency, low mode-number MHD modes (fishbones)}

A quantitative first-principle description of a fishbone pulse from onset to decay still remains a largely open theoretical problem. Progress in this direction would have significant impact on a more general problem of nonlinear description of non-perturbative modes, of which unstable fishbones are an example. The nonlinear interplay between the MHD resonance at the $\mathrm{q}=1$ surface and kinetic energetic particle resonances has been recognized as a critical feature of fishbones. A limited nonlinear modelling [180] has been constructed, in which the only nonlinear effect accounted for is the kinetic response of the energetic particles. The result produces an overall spectral pattern, with a downward frequency sweep, that is similar to the experimental observations. However, the nature of the nonlinearity has been shown to be more complicated. Specifically, the MHD resonance should be more important in the absence of dissipative mechanisms such as resistivity and viscosity at the onset of the nonlinear stage. An initial attempt has been made to understand the nature of the MHD nonlinearity at the early stage of the fishbone pulse near the instability threshold [181], explaining the explosive initial growth of the fishbone. However, considerably more work is needed to be able predict the saturation amplitude of the pulse and to explain the commonly observed frequency sweeping during a pulse decay. Fortunately, as the usual fishbone occurs near the $\mathrm{q}=1$ surface, close to the centre of the machine, the loss of alpha particles from a fishbone is not expected to be severe unless particle orbits are large enough to reach the plasma edge. 


\subsubsection{Kinetic ballooning modes and localized interchange}

Energetic particle effects on higher mode-number kinetic MHD modes, such as Kinetic Ballooning Modes [159] and localized interchange modes [161] have been tackled so far only at an elementary theoretical level. In fact, although the basic understanding of energetic ion interactions with these modes is sound, more detailed analyses enabling quantitative predictions are needed even for the linear regime.

\subsubsection{Alfvén Eigenmodes and phase space nonlinear dynamics}

Near marginal stability, the most efficient nonlinear saturation mechanism for AE is waveparticle trapping, i.e. via phase space nonlinearities [169-170], as confirmed by numerical simulations [182-186]. An accurate theory has been developed to describe the near-threshold nonlinear regimes for weakly unstable Alfvén waves [176-177]. In this case the waves exist in the absence of energetic particles and instability arises when the linear energetic particle drive exceeds the linear damping rate from dissipative processes (see Section 5.5). The resulting spectrum can be steady, pulsating, or chaotic and theory has produced criteria predicting the occurrence of the different regimes. This theory has been applied to interpret JET data [187188]. An excellent agreement has been found in terms of the overall mode behaviour as well as of the dynamical evolution of the spectrum, as shown in Fig. 5.14. At a later nonlinear stage, it is also possible for phase space structures to form [189], whose frequency varies rapidly in the presence of background dissipation. Frequency sweeping signals from this mechanism have been observed in several tokamaks, as shown in Fig. 5.15 in an example extracted from MAST data [190].

A decade ago recurrent bursts of TAEs were observed with neutral beam injection (NBI) in TFTR [191] and DIII-D [192] experiments. Nearly synchronous with these TAE excitations, the neutron emission was observed to drop. It was inferred that the TAE excitation causes a direct loss of the injected beam ions. The relative drop in neutron emission in the TFTR plasma was typically $\sim 10 \%$ (Fig. 4 of Ref. [191]) with a beam confinement time of about onehalf to one-third of the collisional slowing-down time [193]. The TAE activity reduced the beam ion energy confinement time by expelling a substantial fraction of the beam ions before their energy could be transferred to the core plasma through collisions.

TAE bursts and energetic ion loss have been studied theoretically and numerically [194-199]. Simulations based on a reduced MHD method for a configuration typical of the TFTR experiment, which had balanced beam injection, were carried out and the results were reported in Ref. [199]. The simulations reproduced several features of the experiment, including synchronized bursts of multiple TAEs taking place at regular time intervals, the modulation depth in the stored beam energy and a stored beam energy of about one-third of the classical slowing-down distribution. Surface of section plots demonstrated that particle losses are caused both by resonance overlap of different Eigenmodes and by the disappearance of KAM surfaces in phase space due to overlap of higher-order islands created by a single Eigenmode.

However, some discrepancies between the measured and calculated saturation amplitude remain. The simulation mentioned above predicts saturation levels of $\delta B / B \sim 2 \times 10^{-2}$, whereas the estimate from the measured experimental plasma displacement [200] gives $\delta B / B \sim 10^{-3}$. A test particle simulation for the DIII-D experiment also predicted beam ion losses an order of magnitude lower than the observed values [201]. More sophisticated MHD or gyro-kinetic calculations are needed to examine how lower level saturation can be achieved. Detailed experimental measurements of the fast-ion transport and spatial 
eigenfunction are also needed to validate the corresponding numerical simulations.

\subsubsection{Energetic Particle Modes and avalanches}

The nonlinear dynamics of EPMs is regulated by their resonant character and is associated with a redistribution of the energetic ion source [171-174]. The fast ion drive plays a crucial role in determining the radial mode structure [202], which tends to be localized where the drive is the strongest [203]. Fast frequency chirping is expected on time scales shorter than the thermal transport time, due to the nonlinear changes in the fast particle distribution [203$205,126]$. The signature of typical times shorter than equilibrium time scales is similar to that of hole-clump pair creation in phase space near marginal stability [206-207]. In fact, a uniform transition from $\mathrm{AE}$ nonlinear dynamics near marginal stability to macroscopic energetic particle redistribution due to strongly unstable EPMs is expected as the fast ion power density increases.

The EPM excitation threshold corresponds to the condition that the net linear drive (i.e. the difference between growth rate and damping rate) of the kinetic shear Alfvén waves is larger than the characteristic frequency separation of the discretized kinetic shear Alfvén spectrum [202]. Under these conditions, the discretized kinetic shear Alfvén spectrum transforms into the Alfvén continuum. The EPM threshold is then determined by the balance between the drive and the continuum damping. The system is naturally far away from marginal stability of the discrete kinetic shear Alfvén spectrum, for which the continuum damping is not a dominant effect. At the same time, the nonlinear threshold for strong EPM-induced fast particle transport and the linear EPM excitation threshold are strictly connected and generally have similar values [203]. Therefore, both linear and nonlinear EPM dynamics are expected to weakly depend on details of the fast particle distribution function in velocity space, provided that the relevant resonances exist (precession, precession-bounce, transit).

Right above the threshold in energetic particle power density for EPM excitation, fast ions are expected to be radially convected outward via the mode particle pumping mechanism [208]. The secular radial displacement of energetic ions [209] is accompanied by a convective amplification of the EPM mode structure [210], which is predicted to follow the moving source because of the EPM self-trapping at the radial location of maximum drive [211]. This process is depicted in Fig. 5.16 [210], which shows the calculated time evolution of the radial structure of an EPM with toroidal mode number $n=4$ and the deviation, $\delta \alpha_{E}$, of the energetic particle $\alpha_{E} \equiv-R_{0} q^{2}\left(d \beta_{E} / d r\right)$ from the corresponding equilibrium value, due to nonlinear dynamics. Since the EPM drive scales as $\alpha_{E}$, Fig. 5.16 demonstrates the radial propagation of an unstable front, i.e. of an avalanche, which continues until the EPM becomes sub-critical with respect to its resonant excitation condition. After the rapid convection phase, occurring on typical times that scale as the inverse EPM linear growth rate, fast ion transport is expected to continue as a slower diffusive process in the saturated fields [212]. The dynamic picture of Fig. 5.16 is accompanied by a radial fragmentation of the EPM mode structure [213], similar to the modulational instability of drift waves and zonal flow spontaneous generation [214].

In the absence of a self-consistent analysis of energetic particle source formation, accounting for collective plasma behaviours on the Alfvén time scale as well as fast particle Coulomb collisions with the thermal plasma, a simpler, yet important issue to address is the consistency of burning plasma scenarios with resonant EPM excitation. Stated otherwise, one needs to address the question of whether or not the $\alpha_{E}$ profiles obtained from power deposition calculation locally exceed the threshold condition for EPM excitation, $\alpha_{E, c r i t}$, leading to EPM 
induced energetic ion transport.

In the proposed high B-field burning plasma experiments, such as IGNITOR [215] and FIRE [216], $\alpha_{E, \text { crit }}$ significantly exceeds the nominal $\alpha_{E}$ values of reference scenarios [212]. Thus, EPMs are not expected to be an issue on these devices. In ITER-FEAT [217] the situation is less definite. Considering fusion alpha particles only, the nominal value corresponds to $\alpha_{E} \approx 0.65 \alpha_{E, \text { crit }}$ in the conventional shear reference scenario, whereas EPMs are unstable in the reversed shear case [218]. In this scenario, an unstable $n=2$ EPM with $\gamma \approx 0.07 \tau_{A 0}^{-1}$, where $\tau_{A 0}=R_{0} / v_{A}(r=0)$, is predicted to yield a rapid convection of energetic particles, corresponding to a displacement of the maximum of $\alpha_{E}$ from $(r / a)=0.42$ to $(r / a)=0.52$ in $\approx 66 \tau_{A 0}$. The characteristic energetic particle diffusion time in the saturated EPM fields is $\tau_{\text {diff }} \approx 1.6 \times 10^{3} \tau_{A 0}$.

The nonlinear Alfvén mode dynamics gradually changes from the weak nonlinear AE behaviour, due to wave-particle trapping, to the strong nonlinear regime described above and typical of EPM. Dedicated theoretical studies of energetic particle transport in reversed shear scenarios have demonstrated that lower $q_{\min }$ values, more centrally located in the plasma column, and higher $r^{2} q_{\min }^{\prime \prime}$ are preferable and yield smaller EPM induced energetic particle losses.

The theoretical description of nonlinear EPM dynamics is consistent with the experimental observation of Abrupt Large amplitude Events (ALE) [179] on JT-60U. These are Alfvénic oscillations with poloidal amplitude $\delta B_{\theta} / B_{\theta} \sim 10^{-3}$, characteristic time scale of $200 \div 400 \mu \mathrm{s}$ and strong frequency chirping. First reported in Ref. [30], these modes are responsible for a $\leq 15 \%$ drop in the neutron rate with a corresponding increase in the energetic particle loss signal, consistent with the resonance criterion of the mode particle pumping. Experimental measurements also indicate macroscopic fast ion radial redistribution in the plasma core [33], with very similar features to those evidenced in numerical simulations [219].

\subsubsection{Impact on plasma diagnostics}

In burning plasmas there will be a need to control the plasma characteristics externally. Hence the nature of the plasma state will need to be known and it is quite likely that the MHD response induced by energetic particles will produce valuable information for the control of the plasma. Present-day experiments give strong evidence that both linear and nonlinear features of Alfvénic waves driven by energetic particles may add significantly to an understanding of the nature of the discharge. For example, today the presence of so-called Alfvén cascades allows one to infer that the discharge is shear reversed and how fast the minimum q-value is changing.

The emergence of grand cascades (where all the n-numbers appear almost simultaneously) indicates that the minimum q-value is at a rational surface. This knowledge has enabled JET experimentalists to trigger transport barriers in an efficient manner [220]. Another interesting opportunity comes from the theory of frequency sweeping, which indicates that the rate of sweeping is proportional to the internal fields generated by the instability. An attempt to correlate the predicted internal fields with that measured with Mirnov coils in MAST have shown an agreement between the theory and measurement of the internally generated field to within a factor of two [190]. 


\subsection{Summary and Conclusions}

One of the major scientific goals for ITER is to reach and explore the burning plasma regime, in which significant amounts of energy are generated by the D-T fusion reactions. Such a regime is characterised by a large isotropic population of fusion produced alpha particles, born with a largely supra-thermal energy, $3.5 \mathrm{MeV}$, and providing the dominant heating to the plasma.

The physical processes related to energetic ions in burning plasmas are therefore fundamental building blocks for fusion physics both on the way to ITER and during ITER operation itself. A basic understanding from the present generation of experiments is needed to design plasma scenarios in ITER that are compatible with the strong self-heating that defines the burning plasma regime, as well as to constitute the scientific background to correctly collect and interpret the data that will come from ITER discharges reaching this regime.

In the past few years, this problem has been analyzed theoretically and experimentally with increasing attention as the perspective of a burning plasma experiment became more realistic. New theoretical and numerical tools have been developed, and the experiments have taken advantage of new, dedicated active and passive diagnostic methods for energetic particles and the modes interacting with them. In addition, new schemes to create energetic ions simulating the fusion-produced alphas have been introduced. These schemes access experimental conditions of direct relevance for burning plasma operations, in particular in terms of the Alfvénic Mach number and of the normalised pressure gradient of the energetic ions, the free energy source for alpha-driven instabilities.

A limitation of these methods is that orbit characteristics and size, in terms of phase space topology and machine spatial scale, respectively, cannot always match those of ITER or a fusion reactor in the burning regime. For circulating fast ions, negative or positive Neutral Beam Injection provides an efficient tool to study the interaction with waves and the associated ion transport for ITER relevant energy and orbit characteristics. On the other hand, in present experiments, fast ion tails generated by Ion Cyclotron Resonance Heating are generally characterized by large orbits, comparable with the machine size, contrary to the typical conditions of fusion products in burning plasmas. To overcome this, energetic trapped electron populations could provide a complementary tool to study collective mode excitations of the Alfvénic branch by trapped fast particles with small orbits.

In the absence of collective modes, direct losses of fast ions due to imperfections in the toroidal magnetic field, referred to as ripple losses, are now relatively well understood. Enough information is available to optimize ferritic inserts in ITER for a significant reduction of ripple alpha losses in reversed shear configurations. Nevertheless, although classical ripple losses on their own are not expected to provide serious limitations to ITER operations, progress remains to be made on the coupling with the alpha particle redistribution caused by collective instabilities, for all possible classes of orbits.

The role of fast ions on low-frequency MHD modes is essentially well understood, and the important dynamical processes that play a major role in present day experiments are well documented experimentally and modelled theoretically. The nonlinear fast ion interaction with kink and tearing modes is qualitatively understood, but quantitative comparisons need to be further pursued to be able to establish precise predictions. This area is of particular concern 
for ITER, as the stabilisation of sawteeth by fast particles can lead to large internal relaxation events that trigger neoclassical tearing modes, justifying the large number of dedicated studies conducted on many tokamaks during the past few years.

Linear theory provides a rudimentary understanding of kinetic ballooning and localized interchange modes at present, but more detailed analyses enabling quantitative predictions are needed even for the linear regime. A significant effort is expected to take place subsequently to model the corresponding nonlinear evolution.

Following a significant effort in the development of experiments and of the corresponding interpretative models, the main aspects of the linear interaction between energetic ions and high frequency Alfvénic fluctuations, such as Alfvén Eigenmodes (AEs), are qualitatively understood. A large database of experimental results was constructed in either stable or marginally unstable regimes, allowing a direct assessment of the linear stability properties of the modes. Comparisons between experimental measurements of mode structures and drive/damping mechanisms are approaching a satisfactory degree of consistency. Nevertheless, measurements of damping and drive of intermediate and high mode numbers still need to be taken, and a number of non-trivial points remain to be clarified concerning damping rates and mode structures in operation scenarios in which the wave physics depends on the details of equilibrium profiles.

The weakly nonlinear behaviour of Alfvén Eigenmodes is well characterized theoretically and experimentally. By using theoretical predictions of phase space nonlinear dynamics close to marginal stability, it is even possible to extract information on some aspects of the particle phase space distribution from the measured instability spectral features.

On the other hand, the strongly unstable scenarios, dominated by nonlinear dynamical processes and identified in nonlinear numerical simulations of Alfvén Eigenmodes and Energetic Particle Modes, in which fluctuation bursts are accompanied by energetic ion redistribution and losses over short time scales, are much less frequent in present experiments. Experimental conditions with strong heating producing large fractions of energetic ions that drive large amplitude Alfvén Eigenmodes and/or Energetic Particle Modes, leading to measurable fast ion redistribution, need to be identified. Comparisons with more detailed theoretical and numerical analyses will also be needed to assess the potential implications of these phenomena on burning plasma scenarios. In general, more progress is required to precisely assess the impact of collective instabilities of Alfvénic nature on fast ion distributions, both in energy and in radial position, and a fortiori to design possible schemes to use the fast particle-wave interaction as a control tool for the burning plasma. The link with fishbones and bursty MHD activity at lower frequencies should also be assessed.

Redistribution and losses can also be caused by the combined effect of a large number of modes simultaneously driven unstable by the fast ions. Although this is a unique feature of large scale burning plasma experiments, which are characterised by a large number of fast ion orbits contained within the plasma radius, the possibility of producing experimental conditions that approach this situation in present devices should be explored, in parallel with nonlinear numerical simulations.

An important problem that still represents a challenge for formal theoretical analyses as well as for numerical computations is that of the interaction between the background plasma turbulence and energetic ions. Limited experimental efforts have been devoted to study such 
problem, while modern nonlinear dynamics methods have been applied so far only to look at test particle transport in oversimplified situations.

Present day experiments, theory and numerical modelling tools appear to have the necessary prerequisites to lead to significant progress in the field of energetic ion physics in the next few years. However, entire new classes of problems are expected to emerge in a burning plasma, related to its very nature as a complex self-organized system. The different aspects of the dynamics of such system are all coupled, with for example the plasma turbulence influencing the thermal plasma and the energetic ion profile, the energetic ions driving collective modes and being influenced by them, the fusion power density being determined by the self-heating provided by the energetic ions, and the plasma power density, in turn, determining the character of the turbulence. The investigation of these new problems, related to the basic science of complex systems and potentially having a dramatic impact on the plasma fusion performance, will only be possible in an actual burning plasma experiment such as ITER and will constitute a greatly exciting task for both experimental and theoretical plasma physics. 


\section{References}

[1] Heidbrink W.W. and Sadler G.J., Nucl. Fusion 34 (1994) 535.

[2] ITER Physics Expert Group on Energetic Particles, Heating, and Current Drive et al., Nucl. Fusion 39 (1999) 2471.

[3] ITER Physics Expert Group on Energetic Particles, Heating, and Current Drive et al., Nucl. Fusion 39 (1999) 2495.

[4] Jassby D.L., Strachan J.D., Bell M.G., et al., Nucl. Fusion 39 (1999) 189.

[5] Ruskov E., Budny R.V., McCune D.C., et al., Phys. Rev. Lett. 82 (1999) 924.

[6] Fu G.Y., Nazikian R., Bidny R. and Chang Z., Phys. Plasmas 5 (1998) 4284.

[7] Stratton B.C., Budny R.V., Darrow D.S., et al., Nucl. Fusion 39 (1999) 1309.

[8] Zweben S.J., Darrow D.S., Fredrickson E.D., et al., Nucl. Fusion 39 (1999) 1097.

[9] Zweben S.J., Budny R.V., Darrow, D.S., et al., Nucl. Fusion 40 (2000) 91.

[10] Källne J., Ballabio L., Frenje J. et al., Phys. Rev. Lett. 85 (2000) 1246.

[11] Korotkov A.A., Gondhalekar A., and Akers R.J., Phys. Plasmas 7 (2000) 957.

[12] Mantsinen M.J., Jarvis O.N., Kiptily V.G., et al., Nucl. Fusion 41 (2001) 1815.

[13] Henriksson H., Conroy S., Ericsson G., et al., Plasma Phys. Controlled Fusion 44 (2002) 1253.

[14] Tardocchi M., Conroy S., Ericsson G., et al., Nucl. Fusion 42 (2002) 1273.

[15] Eriksson L.-G., Mantsinen M.J., Rimini F.G., et al., Nucl. Fusion 38 (1998) 265.

[16] Heidbrink W.W., Fredrickson E.D., Mau T.K., et al., Nucl. Fusion 39 (1999) 1369.

[17] Pinsker R.I., Baity F.W., Bernabei S.N., et al., in Radio Frequency Power in Plasmas

(Proc. 13th International Conference (Annapolis, 1999), p. 144, New York, 1999, AIP.

[18] Petty C.C., Baity F.W., Degrassie J.S., et al., Plasma Phys. Controlled Fusion 43 (2001) 1747.

[19] Mantsinen M.J., Mayoral M.-L., Kiptily V.G., et al., Phys. Rev. Lett. 88 (2002) 105002.

[20] Mantsinen M.J., Petty C.C., Eriksson L.-G., et al., Phys. Plasmas 9 (2002) 1318.

[21] Eriksson L.-G., Mantsinen M., Borba, D., et al., Phys. Rev. Lett. 81 (1998) 1231.

[22] Mantsinen M.J., Eriksson L.-G., Gormezano, C., et al., Nucl. Fusion 40 (2000) 1773.

[23] Mantsinen M.J., Ingesson L.C., Johnson T., et al., Phys. Rev. Lett. 89 (2002) 115004.

[24] Kiptily V.G., Cecil F.E., et al., Nucl. Fusion 42 (2002) 999.

[25] Wong K.-L., and Ono M., Nucl. Fusion 24 (1984) 615.

[26] Testa D., Lashmore-Davies C.N., Gondhalekar A., et al., Plasma Phys. Controlled Fusion 41 (1999) 507.

[27] Suzuki S., Shirai T., Nemoto M., et al., Plasma Phys. Controlled Fusion 40 (1998) 2097.

[28] Tobita K. and The JT-60 Team, Plasma Phys. Controlled Fusion 41 (1999) A333.

[29] Kusama Y., Fu G.Y., Kramer G.J., et al., Plasma Phys. Controlled Fusion 41 (1999) 1167.

[30] Kusama Y. et al., Nucl. Fusion 39 (1999) 1837.

[31] Oikawa T., Ushigusa K., Forest C.B., et al., Nucl. Fusion 40 (2000) 435.

[32] Oikawa T., Kamada Y., Isayama A., et al., Nucl. Fusion 41 (2001) 1575.

[33] Shinohara K. et al., Nucl. Fusion 42 (2002) 942.

[34] McClements K.G., Gryaznevich M.P., Sharapov S.E., et al., Plasma Phys. Controlled Fusion 41 (1999) 661.

[35] Gryaznevich M.P. and Sharapov S.E., Plasma Phys. Controlled Fusion 46 (2004) S15.

[36] Fredrickson E.D., Gorelenkov N., Cheng C.Z., et al., Phys. Rev. Lett. 87 (2001) 145001.

[37] Fredrickson E.D., Gorelenkov N., et al., Phys. Plasmas 9 (2002) 2069.

[38] Akers R.J., Appel L.C., Carolan P.G., et al., Nucl. Fusion 42 (2002) 122.

[39] Gorelenkov N. N., Cheng C.Z., et al., Nucl. Fusion 42 (2002) 977.

[40] Fredrickson E.D., Cheng C.Z., Darrow D., et al., Phys. Plasmas 10 (2003) 2852. 
[41] Fredrickson E., Chen L., and White R., Nucl. Fusion 43 (2003) 1258.

[42] Gorelenkov N.N., Belova E., Berk H.L., et al., Phys. Plasmas 11 (2004) 2586.

[43] Heidbrink, W.W., Fredrickson E., Gorelenkov N.N., et al., Plasma Phys. Controlled

Fusion 45 (2003) 983.

[44] Suborov E.V., Holzhauer E., Kasparek W., et al., Nucl. Fusion 38 (1998) 661.

[45] Teo C.-Y., Weller A., Konrad C., et al., Nucl. Fusion 38 (1998) 409.

[46] Toi K., Takechi M., Isobe M., et al., Nucl. Fusion 40 (2000) 1349.

[47] Toi K. Yamamoto S., Nakajima N., et al., Plasma Phys. Controlled Fusion 46 (2004) S1.

[48] Baldzuhn J., Werner A., Wobig H., et al., Plasma Phys. Controlled Fusion 45 (2003) 891.

[49] Shalashov A.G., Suvorov E.V., Lubyako L.V., et al., Plasma Phys. Controlled Fusion 45 (2003) 395.

[50] Weller A., Anton M., Geiger J., et al., Phys. Plasmas 8 (2001) 931.

[51] Budny R.V., Nucl. Fusion 42 (2002) 1383.

[52] Putvinski, S.V, Nucl. Fusion 38 (1998) 1275.

[53] Tobita, K., et al., Nucl. Fusion 35 (1995) 1585.

[54] Zweben, S.J., et al., Nucl. Fusion 38 (1998) 739.

[55] Hawryluk R.J., Batha S., Blanchard W., et al., Phys. Plasmas 5 (1998) 1577.

[56] Kawashima, H., et al., Nucl. Fusion 41 (2001) 257.

[57] Sato, M., et al., Nucl. Fusion 42 (2002) 1008.

[58] Shinohara, K., et al., Nucl. Fusion 43 (2003) 586.

[59] Basiuk, V., et al., Nucl Fusion 44 (2004) 181.

[60] Assas, S., et al., Plasma Phys. Control. Fusion 45 (2003) 145.

[61] Goldston, R.J., White R.B., Boozer, A.H., Phys. Rev. Lett. 47 (1981) 647.

[62] Tobita, K., et al., Nucl. Fusion 37 (1997) 1583.

[63] Tobita, K., Ozeki T., Nakamura Y., Plasma Phys. Control. Fusion 46 (2004) S95.

[64] Goloborod'ko V.Ya., Kolesnichenko Ya.I., and Yavorskij V.A., Physica Scripta T 16 (1987) 46.

[65] Tani K.,et al., J. Phys. Soc. Japan 50 (1981) 1726.

[66] Konovalov S.V. et al., JAERI-Research 94-033 (1993).

[67] Konovalov S.V., et al., 28th EPS Conference on Contr. Fusion and Plasma Phys.

Funchal, 18-22 June 2001 ECA Vol. 25A (2001) 613-616.

[68] Tobita, K., et al., Plasma Phys. Control. Fusion 45 (2003) 133.

[69] Fisch N.J., Nucl. Fusion 40 (2000) 1095.

[70] Angioni C., Pochelon A., Gorelenkov N.N., et al., Plasma Phys. and Contr. Fus. 44 (2002) 205.

[71] Kramer G.J., et al., Nucl. Fusion 40 (2000) 1383.

[72] Nave M.F.F., Gorelenkov N.N., McClements K.G., et al., Nucl. Fusion 42 (2002) 281.

[73] Bombarda F., Bonoli P.T., Coppi B., et al., Nucl. Fusion 38 (1998) 1861.

[74] Bernabei S., Bell M.G., Budny R., Darrow D., Fredrickson E.D. et al., Phys. Plasmas 6 (1999) 1880.

[75] Bernabei S., Budny R.V., Fredrickson E.D., et al., Nucl. Fusion 41 (2001) 513.

[76] Kolesnichenko Y.I., Lutsenko V.V., White R.B., Yakovenko Y.V., and Zweben S.J., Phys. Plasmas 6 (1999) 1117.

[77] Kolesnichenko Y.I., Lutsenko V.V., White R.B., and Yakovenko Y.V., Nuclear Fusion 40 (2000) 1325.

[78] Helander P., Eriksson L.-G., Akers R.J., Byrom C., Gimblett C.G., et al., Phys. Rev. Letters 89 (2002) 235002-1.

[79] Petrov M.P., Bell R., Budny R.V., Gorelenkov N.N., Medley S.S., et al., Phys. Plasmas 6 (1999) 2430.

[80] Kramer G.J., Cheng C.Z., Kusama Y., Nazikian R., Takeji S., et al., Nucl. Fusion 41 
(2001) 1135.

[81] Kolesnichenko, Y.I. and Yakovenko, Y.V., Nucl. Fusion 36 (1996) 159.

[82] Fredrickson E.D., Phys. Plasmas 9 (2002) 548.

[83] Guenter S., Gude A., Koslowski H.R., et al., Nucl. Fusion 39 (1999) 1793.

[84] Carolipio E.M., Heidbrink W.W., Forest C.B., and White R.B., Nucl. Fusion 42 (2002) 853.

[85] Mantsinen M., Angioni C., Eriksson L.-G., Gondhalekar A., Hellsten T., et al., Plasma Phys. Control. Fusion 44 (2002) 1521.

[86] Valovic M., Lloyd B., McClements K.G., et al., Nucl. Fusion 40 (2000) 1569.

[87] Sauter O., Westerhof E., Mayoral M.L., et al., Phys. Rev. Letters 88 (2002) 105001.

[88] Mantsinen M., Sharapov S., Alper B., Gondhalekar A., and McDonald D.C., Plasma Phys. and Contr. Fusion 42 (2000) 1291.

[89] Gormezano C., Baranov Y.F., Challis C.D., et al., Phys. Rev. Letters 80 (1998) 5544.

[90] Efthimion P.C., von Goeler S., Houlberg W.A., et al., Phys. Plasmas 5 (1998) 1832.

[91] Luce T.C. et al, Nucl. Fusion 41 (2001) 1585.

[92] Horton L.D., Sartori R., Balet B., et al., Nucl. Fusion 39 (1999) 993.

[93] Heidbrink W.W., Ruskov E., Carolipio E.M., Fang J., van Zeeland M.A., et al., Phys. Plasmas 6 (1999) 1147.

[94] Kass T., Bosch H.-S., Hoenen F., et al., Nucl. Fusion 38 (1998) 807.

[95] McClements K.G., Gryaznevich M.P., Sharapov S.E., Akers R.J., Appel L.C. et al., Plasma Phys. and Contr. Fusion 41 (1999) 661.

[96] Toi K., Takechi M., Takagi S., et al., Nucl. Fusion 39 (1999) 1929.

[97] Guenter S., et al, Nucl. Fusion 39 (1999) 1535.

[98] Sesnic S., Gunter S., Gude A., et al., Phys. Plasmas 7 (2000) 935.

[99] Borba D., Alper B., Budny R.V., et al., Nucl. Fusion 40 (2000) 775.

[100] Hu B, Betti R, Manickam J, Phys. Plasmas 12 (2005) 057301.

[101] Mantsinen M.J., Eriksson L.-G., Gondhalekar A., and Hellsten T., Nucl. Fusion 39 (1999) 459.

[102] Mantsinen M., Eriksson L.-G., Gauthier E., Hoang G.T., Joffrin E. et al., Plasma Phys. Control. Fusion 45 (2003) A445-A456.

[103] Eriksson L.-G., Mantsinen M., Borba D., Fasoli A., et al., Phys. Rev. Lett. 81 (1998) 1231.

[104] Mantsinen M., Ingesson L.C., Johnson T., Kiptily V., Mayoral M.-L., et al., Phys. Rev. Letters 89 (2002) 115004-1.

[105] Zastrow K.-D., Core W.G.F., Eriksson L.-G., et al., Nucl. Fusion 38 (1998) 257.

[106] Noterdaeme J.-M., Budny R., Cardinali A., Castaldo C., Cesario R., et al., Nuclear Fusion 43 (2003) 202.

[107] Fasoli A., et al., Phys. Plasmas 5 (2000) 1816.

[108] Todo Y., Berk H. L., Breizman B. N., Phys. Plasmas 10 (2003) 2888.

[109] Fu G.Y., Cheng C.Z., Phys. Fluids B 4 (1992) 3722.

[110] Breizman B.N. and Sharapov S..E., Plasma Physics Contr. Fusion 37 (1995) 1057.

[111] Gorelenkov, N.N., et.al., Nuclear Fusion 43 (2003) 594.

[112] Candy J., Plasma Phys. Controlled Fusion 38 (1996) 795.

[113] Jaun A., Appert K., Vaclavik J., Villard L., Comput. Phys. Commun. 92 (1995) 153.

[114] Borba D. and Kerner W., J. Comput. Phys. 153 (1999) 101

[115] Gorelenkov N.N., Sharapov S., Physica Scripta 45 (1991) 163.

[116] Fu G.Y., Cheng C.Z., et.al. Phys. Plasmas 3 (1996) 4036.

[117] Jaun A., Fasoli A., Heidbrink W.W., Phys. Plasmas 5 (1998) 2952.

[118] Jaun A., Fasoli A., Vaclavik J., Villard, L., Nucl. Fusion 40 (2000) 1343.

[119] Borba D., Berk H.L., Breizman B.N., Fasoli A., Nabais F., Pinches S.D., Sharapov 
S.E., Testa D. and JET-EFDA contributors, Nucl. Fusion 42 (2002) 1029.

[120] Fu G.Y., Berk H.L., and Pletzer A., Phys. Plasmas 12 (2005) 082505.

[121] Lauber Ph., Günter S. and Pinches S.D., Phys. Plasmas 12 (2005) 122501.

[122] Heidbrink W.W., et al., Phys. Plasmas 4 (1997) 3663.

[123] Heidbrink W.W., et al., Phys. Plasmas 6 (1999) 1147.

[124] Gorelenkov N.N., Cheng C.Z., Tang W.M., Phys. Plasmas 5 (1998) 3389.

[125] Heidbrink W.W., Gorelenkov,N.N., Murakami M., Nucl. Fusion 42 (2002) 972.

[126] Gorelenkov N.N., Heidbrink W.W., Nucl. Fusion 42 (2002) 150.

[127] Zonca F., et al., Phys. Plasmas 6 (1999) 1917.

[128] Briguglio S., et al, Nucl. Fusion 40 (2000) 701.

[129] Jaun A., et al., in Fusion Energy 2002 (Proc. 19 ${ }^{\text {th }}$ Int. Conf. Lyon, 2002) (Vienna:

IAEA) CD-ROM CT/P-06 file and

http://www.iaea.org/programmes/ripc/physics/fec2002/html/fec2002.htm.

[130] Sharapov S.E., Alper B., Berk H.L., Phys. Plasmas 9 (2002) 2027.

[131] Breizman B.N., et al., Phys. Plasmas 10 (2003) 3649.

[132] Zonca F., et al., Phys. Plasmas 9 (2002) 4939.

[133] Kramer G.J., et al., Plasma Physics Controlled Fusion 46 (2004) L23.

[134] Zonca F., Chen L., Phys. Plasmas 7 (2000) 4600.

[135] Takechi M., et al., Phys. Plasmas 12 (2005) 082509

[136] Fukuyama A., Akutsu T., in Fusion Energy 2002 (Proc. 19 ${ }^{\text {th }}$ Int. Conf. Lyon, 2002)

(Vienna: IAEA) CD-ROM TH/P3-14 and

http://www.iaea.org/programmes/ripc/physics/fec2002/html/fec2002.htm.

[137] Nazikian R., et al.. Phys. Rev. Lett. 91 (2003) 125003.

[138] Nazikian R., et al., Phys. Rev. Lett. 78 (1997) 2976.

[139] Nazikian R., et al., Physics Plasmas 5 (1998) 1703.

[140] Sharapov S.E., et al., Phys. Rev. Lett. 93 (2004) 165001.

[141] Snipes J.A, et al., Plasma Physics Contr. Fusion 42 (2000) 381.

[142] Nazikian R. et al, in Fusion Energy 2004 (Proc. 20 ${ }^{\text {th }}$ Int. Conf. Vilamoura, 2004)

(Vienna: IAEA) CD-ROM EX/5-1 and

http://www-naweb.iaea.org/napc/physics/fec/fec2004/datasets/index.html.

[143] Van Zeeland M., et al., Plasma Phys. Control. Fusion 47 (2005) L31.

[144] Fasoli A., et al., Phys. Rev. Lett. 75 (1995), 645.

[145] Fasoli A., et al., Phys.Plasmas 7 (2000) 1816.

[146] Cheng C.Z., Phys. Rep. 211 (1992) 1.

[147] Testa D., Fasoli A., et al., Nucl. Fusion 41 (2001) 809.

[148] Testa D., et al., Plasma Phys. Control. Fusion 46 (2004) S59.

[149] Fasoli A., Jaun A., Testa D., Phys. Lett. A 265 (2000) 288.

[150] Testa D., et al., Nucl. Fusion 43 (2003) 479.

[151] Testa D., Fasoli A., Jaun A., et al., Nucl. Fusion 43 (2003) 724.

[152] Jaun A., et al., Plasma Phys. Control. Fusion 39 (1997) 549.

[153] Heidbrink W.W., et al., Plasma Phys. Control. Fusion 45 (2003) 983.

[154] Murari A., Bertalot L., Bonheure G., et al., Plasma Phys. Contr. Fusion 47 (2005) B249.

[155] Snipes J., et al., Phys. of Plasmas 12 (2005) 056102.

[156] Chen L., White R.B., and Rosenbluth M.N., Phys. Rev. Lett. 52 (1984) 1122.

[157] Coppi B., and Porcelli F., Phys. Rev. Lett. 57 (1986) 2272.

[158] McGuire K., Goldston R., Bell M., et al., Phys. Rev. Lett. 50 (1983) 891.

[159] Tsai S.T., and Chen L., Phys. Fluids B 5 (1993) 3284.

[160] Porcelli F., and Rosenbluth M.N., Plasma Phys. Control. Fusion 40 (1998) 481.

[161] Zonca F., Chen L., Dong J.Q., and Santoro R.A., Phys. Plasmas 6 (1999) 1917.

[162] Rosenbluth M.N., and Rutherford P.H., Phys. Rev. Lett. 34 (1975) 1428. 
[163] Mikhailovskii A.B., Sov. Phys. JETP 41 (1975) 890.

[164] Chen L., 1988 Theory of Fusion Plasmas (Editrice Compositori, Bologna, 1989) p 327.

[165] Fu G.Y., and Van Dam J.W., Phys. Fluids B 1 (1989) 1949.

[166] Biglari H., Chen L., and Zonca F., Phys. Fluids B 4 (1992) 2385.

[167] Cheng C.Z., Chen L., and Chance M.S., 1985 Ann. Phys. (NY) 16121.

[168] Chen L., Phys. Plasmas 1 (1994) 1519.

[169] Berk H.L., and Breizman B.N., Phys. Fluids B 2 (1990) 2246.

[170] Berk H.L., Breizman B.N., and Ye H., Phys. Rev. Lett. 68 (1992) 3563.

[171] Cheng C.Z., Gorelenkov N.N., and Hsu C.T., Nucl. Fusion 35 (1995) 1639.

[172] Vlad G., Zonca F., and Romanelli F., Nucl. Fusion 35 (1995) 1651.

[173] Chen L., and Zonca F., Physica Scripta T60 (1995) 81.

[174] Briguglio S., Zonca F., and Vlad G., Phys. Plasmas 5 (1998) 3287.

[175] Zonca F., Briguglio S., Fogaccia G., and Vlad G., 1999 AIP Conf. Proc. 485 (AIP, New York, 1999) p 56.

[176] Berk H.L., Breizman B.N., and Pekker M., Phys. Rev. Lett. 76 (1996) 1256.

[177] Breizman B.N., Berk H.L., Pekker M., Porcelli F., Stupakov G.V., and Wong K.L.,

Phys. Plasmas 4 (1997) 1559.

[178] Wong K.-L., Plasma Phys. Control. Fusion 41 (1999) R1.

[179] Shinohara K., Kusama Y., Takechi M., et al., Nucl. Fusion 41 (2001) 603.

[180] Candy J., Berk H.L., Breizman B.N., Procelli F., Physics of Plasmas 6 (1999)1822.

[181] Ödblom A., Breizman B.N., Sharapov S.E., Hender T.C., Pastukhov V. P., Physics of Plasmas 9 (2002) 155.

[182] Wu Y., and White R.B., Phys. Plasmas 1 (1994) 2733.

[183] Fu G.Y, and Park W., Phys. Rev. Lett. 74 (1995) 1594

[184] Todo Y., Sato T., Watanabe K., Watanabe T.H., and Horiuchi R., Phys. Plasmas 2

(1995) 2711.

[185] Wu Y., White R.B., Chen Y., and Rosenbluth M.N., Phys. Plasmas 2 (1995) 4555.

[186] Candy J., Borba D., Berk H.L., Huysmans G.T.A., Kerner W., Phys. Plasmas 4 (1997) 2597.

[187] Fasoli A., Breizman B.N., Borba D., Heeter R.F., Pekker M.S., and Sharapov S.E., Phys. Rev. Lett. 81 (1998) 5564.

[188] Heeter R.F., Fasoli A.F., Sharapov S.E., Phys. Rev. Lett. 85 (2000) 3177.

[189] Berk H.L., Breizman B.N., Candy J., Pekker M.S., and Petviashvili N.V., Phys. Plasmas 6 (1999) 3102.

[190] Pinches S.D., Berk H.L., Gryaznevich M.P., Sharapov S.E., and JET-EFDA

Contributors, Plasma Phys. Control. Fusion 46 (2004) S47.

[191] Wong K.L., Fonck R.J., Paul S.F., et al., Phys. Rev. Lett. 66 (1991) 1874.

[192] Heidbrink W.W., Strait E.J., Doyle E., Sager G., and Snider R.T., Nucl. Fusion 31 (1991) 1635.

[193] Wong K.L., Durst R., Fonck R.J., et al., Phys. Fluids B 4 (1992) 2122.

[194] Heidbrink W.W., Duong H.H., Manson J., et al., Phys. Fluids B 5 (1993) 2176.

[195] Berk H.L., Breizman B.N., Fitzpatrick J., and Wong H.V., Nucl. Fusion 35 (1995) 1661.

[196] Berk H.L., Breizman B.N., and Pekker M.S., Nucl. Fusion 35 (1995) 1713.

[197] Berk H.L. and Breizman B.N., 1998 Theory of Fusion Plasmas (Società Italiana di Fisica, Bologna, 1999) p 283.

[198] Todo Y., Watanabe T.-H., Park H.-B., and Sato T., Nucl. Fusion 41 (2001) 1153.

[199] Todo Y., Berk H.L., and Breizman B.N., Phys. Plasmas 10 (2003) 2888.

[200] Durst R.D., Fonck R.J., Wong K.L., et al., Phys Fluids B 4 (1992) 3707.

[201] Carolipio E.M., Heidbrink W.W., Cheng C.Z., et al., Phys. Plasmas 8 (2001) 3391. 
[202] Zonca F., and Chen L., Phys. Plasmas 3 (1996) 323.

[203] Zonca F., and Chen L., Phys. Plasmas 7 (2000) 4600.

[204] Briguglio S., Kar C., Romanelli F., Vlad G., and Zonca F., Plasma Phys. Control. Fusion 37 (1995) A279.

[205] Gorelenkov N.N., et al., Nucl. Fusion 40 (2000)1311.

[206] Berk H.L., Breizman B.N., and Petviashvili N.V., Phys. Lett. A 234 (1997) 213.

[207] Todo Y., Shinohara K., Takechi M., and Ishikawa M., J. Plasma Fus. Res. 79 (2003) 1107.

[208] White R.B., Goldston R.J., McGuire K., Boozer A.H., Monticello D.A., and Park W., Phys. Fluids 26 (1983) 2958.

[209] Briguglio S., Vlad G., Zonca F., and Fogaccia G., Phys. Lett. A 302 (2002) 308.

[210] Zonca F., Briguglio S., Chen L., et al., in Fusion Energy 2002 (Proc. 19th Int. Conf. Lyon, 2002) (Vienna: IAEA) CD-ROM TH/4-4 and

http://www.iaea.org/programmes/ripc/physics/fec2002/html/fec2002.htm.

[211] Zonca F., Briguglio S., Chen L., Dettrick S., Fogaccia G., Testa D., and Vlad G., Phys. Plasmas 9 (2002) 4936.

[212] Vlad G., Briguglio S., Fogaccia G., and Zonca F., Plasma Phys. Control. Fusion 46 (2004) S81.

[213] Zonca F., Briguglio S., Chen L., Fogaccia G., and Vlad G., 2000 Theory of Fusion Plasmas (Società Italiana di Fisica, Bologna, 2000) p 17.

[214] Chen L., Lin Z., and White R.B., Phys. Plasmas 7 (2000) 3129.

[215] Coppi B., Airoldi A., Bombarda F., Cenacchi G., Detragiache P., Ferro C., Maggiora

R., Sugiyama L.E. and Vecchi G., 1999 Ignitor Report MIT RLE PTP 99/06.

[216] Meade D., Comments on Pl. Phys. and Contr. Fus. 2 (2000) 81.

[217] Aymar R., et al., in Fusion Energy 2000 (Proc. 18th Int. Conf. Sorrento, 2000) (Vienna:

IAEA) CD-ROM ITEROV/1 and

http://www.iaea.org/programmes/ripc/physics/fec2000/html/node1.htm.

[218] Polevoi A.R., Medvedev S.Y., Mukhovatov V.S., Kukushkin A.S., Murakami Y.,

Shimada M., and Ivanov A.A., J. Plasma Fusion Res. SERIES 5 (2002) 82.

[219] Vlad G., Briguglio S., Fogaccia G., and Zonca F., 2002 ECA 26B P-4.088.

[220] Joffrin, E. Challis C.D., Conway G.D., Garbet X., Gude A., et al, Nucl. Fusion 43

(2003) 1167. 


\section{Tables and Figures}

Table 5.1 Fast-ion parameters in contemporary experiments compared with projected ITER values.

\begin{tabular}{llllll}
\hline $\begin{array}{l}\text { Tokamak } \\
\text { Fast ion }\end{array}$ & $\begin{array}{l}\text { TFTR } \\
\text { Alpha }\end{array}$ & $\begin{array}{l}\text { JET } \\
\text { Alpha }\end{array}$ & $\begin{array}{l}\text { JT-60U } \\
\text { Deuteriu } \\
\mathrm{m}\end{array}$ & $\begin{array}{l}\text { JET } \\
\text { Alpha }\end{array}$ & $\begin{array}{l}\text { ITER } \\
\text { Alpha }\end{array}$ \\
Source & Fusion & Fusion & Co NBI & $\begin{array}{l}\text { ICRF } \\
\text { tail }\end{array}$ & Fusion \\
Reference & {$[2]$} & {$[2]$} & {$[33]$} & {$[19,51]$} & {$[51]$} \\
\hline$\tau_{S}(\mathrm{~s})$ & 0.5 & 1.0 & 0.085 & 0.4 & 0.8 \\
$\delta / a^{(\mathrm{a})}$ & 0.3 & 0.36 & 0.34 & 0.35 & 0.05 \\
$P_{f}(0)\left(\mathrm{MW} / \mathrm{m}^{3}\right)$ & 0.28 & 0.12 & 0.12 & 0.5 & 0.55 \\
$n_{f}(0) / n_{e}(0)(\%)$ & 0.3 & 0.44 & 2 & 1.5 & 0.85 \\
$\beta_{f}(0)(\%)$ & 0.26 & 0.7 & 0.6 & 3 & 1.2 \\
$\left\langle\beta_{f}\right\rangle(\%)$ & 0.03 & 0.12 & 0.15 & 0.3 & 0.3 \\
max $\left|R \nabla \beta_{f}\right|(\%)$ & 2.0 & 3.5 & 6 & 5 & 3.8 \\
$v_{f}(0) / v_{A}(0)$ & 1.6 & 1.6 & 1.9 & 1.3 & 1.9 \\
\hline (a) Orbit shift from magnetic flux surface for banana particles: $\delta=$ \\
$q p_{f} \sqrt{R / r}$. Note that the particle shift is smaller for passing-particle \\
populations arising from tangential injection.
\end{tabular}



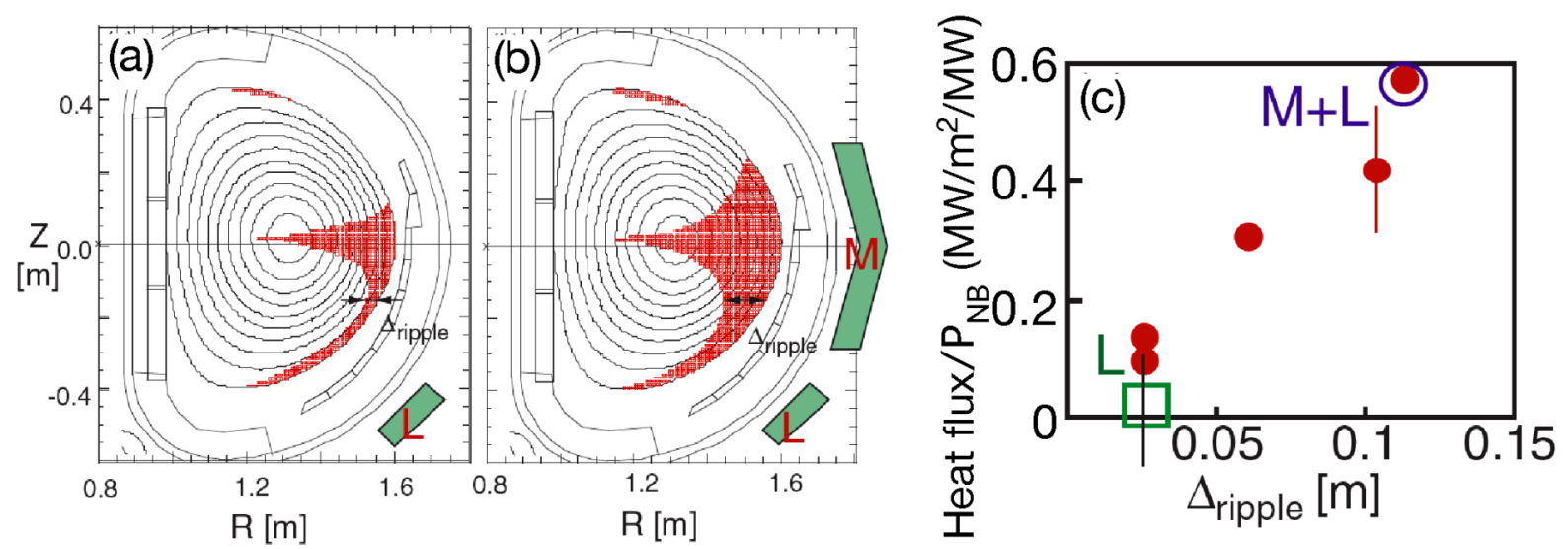

Fig. 5.1 Localized ripple well produced in JFT-2M by FS insert of (a) L and of (b) L+M. And the resulting local ripple-trapped of of NB ions: open square - measured loss for L; open circle - measured for L+M; and closed circles — calculated loss [58]. 
(a)

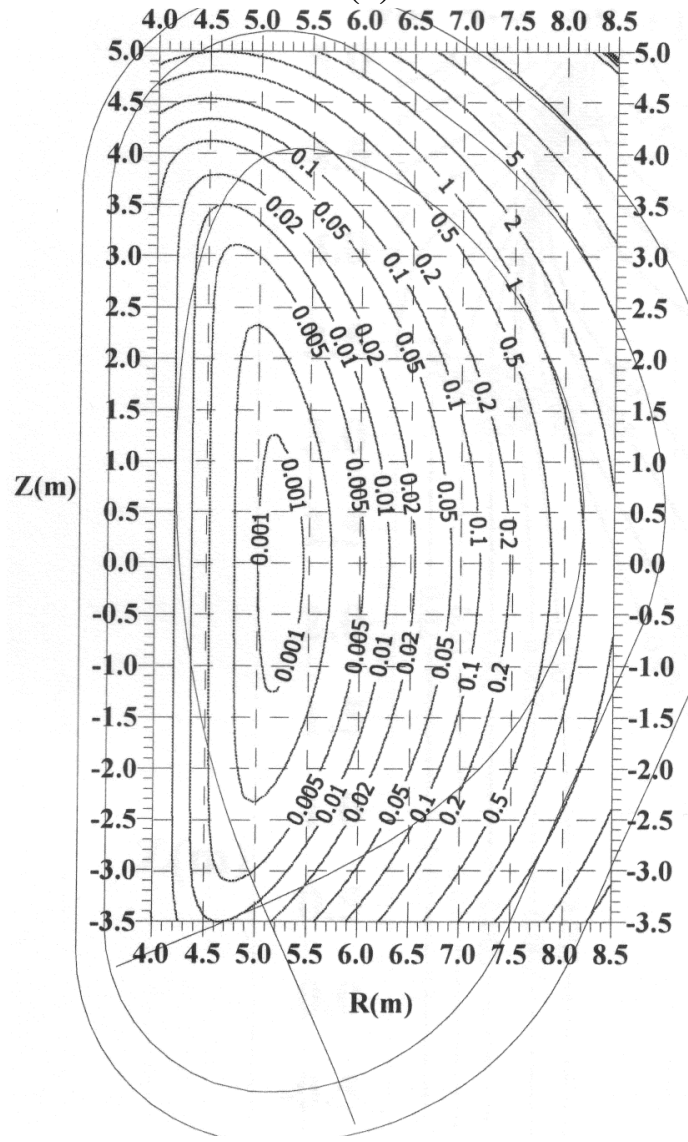

(b)

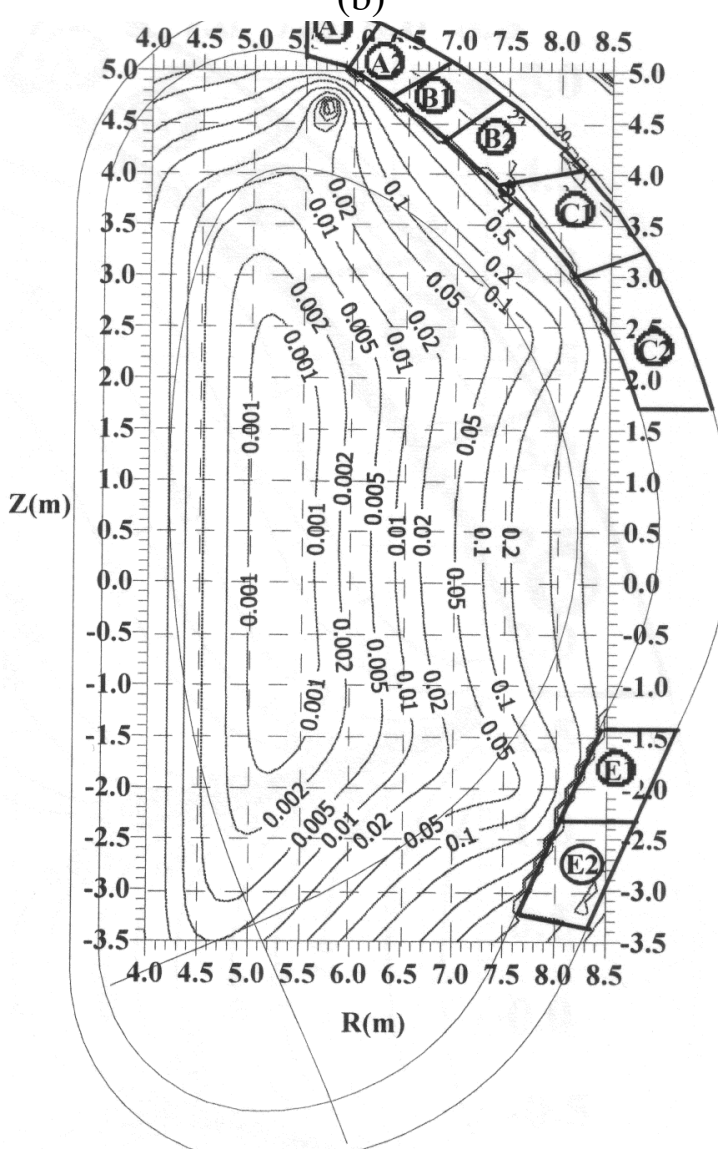

Fig. 5.2 Ripple amplitude contours for (a) TF coils only and (b) TF coils and FS inserts of ITER at $\mathrm{B}_{\mathrm{T}}=5.3 \mathrm{~T}$ [67]. 
(a) No FS inserts

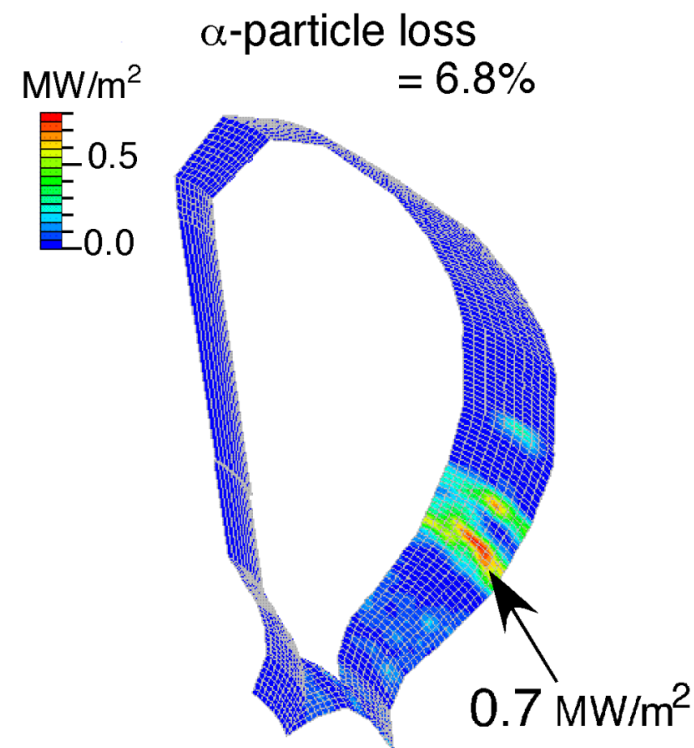

(b) With FS inserts

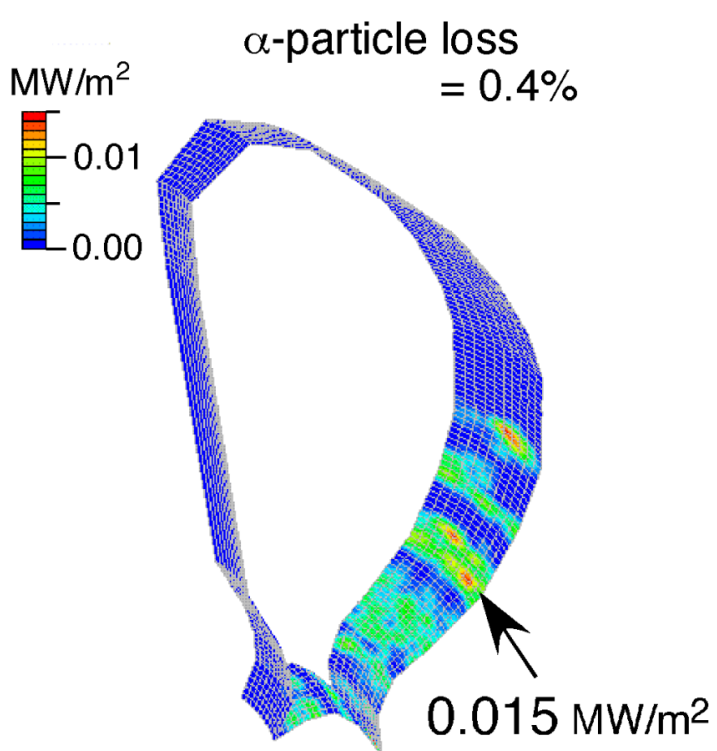

Fig. 5.3 Calculated heat deposition on the ITER first wall for (a) the original TF ripple without FS inserts, and (b) the reduced ripple with the optimized FS inserts [68]. The calculation was done for the reversed shear plasma with $\mathrm{q}_{\min }=2$. 

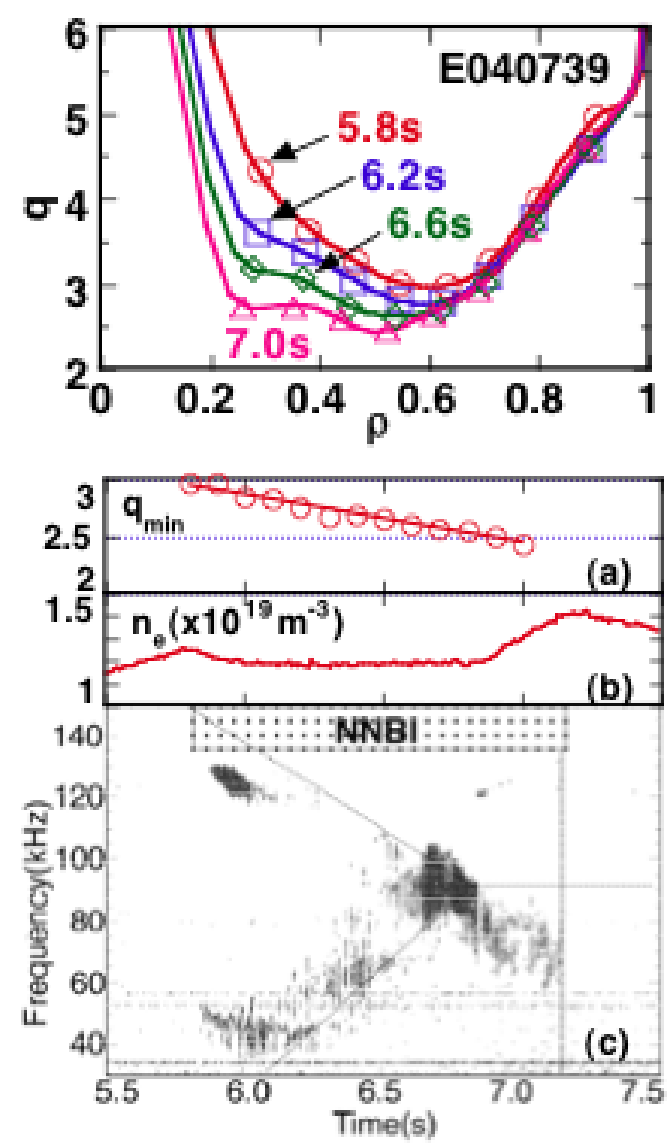

Fig. 5.4 Upper and lower reversed shear Alfvén Eigenmode (RSAE) and toroidal Alfvén Eigenmode (TAE) measured in JT-60U. (a) Temporal evolution of the q-profile measured with MSE. (b) Temporal evolution of $\mathrm{q}_{\min }$, (c) line averaged electron density, (d) a time trace of frequency spectrum of the $\mathrm{n}=1$ instability. 


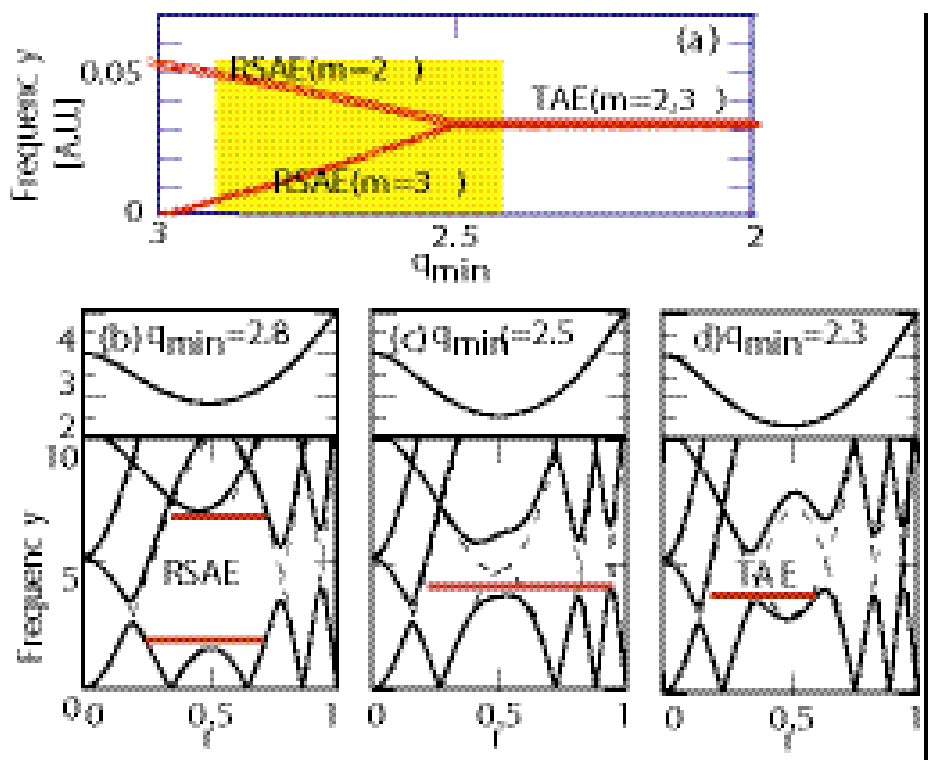

Fig. 5.5 Schematic interpretation of the evolution shown in the previous figure. (a) Frequencies of the RSAE and TAE modes as a function of $\mathrm{q}_{\min }$. Alfvén continuum for $\mathrm{n}=1$ in the reversed shear plasma with (b) $\mathrm{q}_{\min }=2.8$ (c) $\mathrm{q}_{\min }=2.5$ and (d) $\mathrm{q}_{\min }=2.3$. 


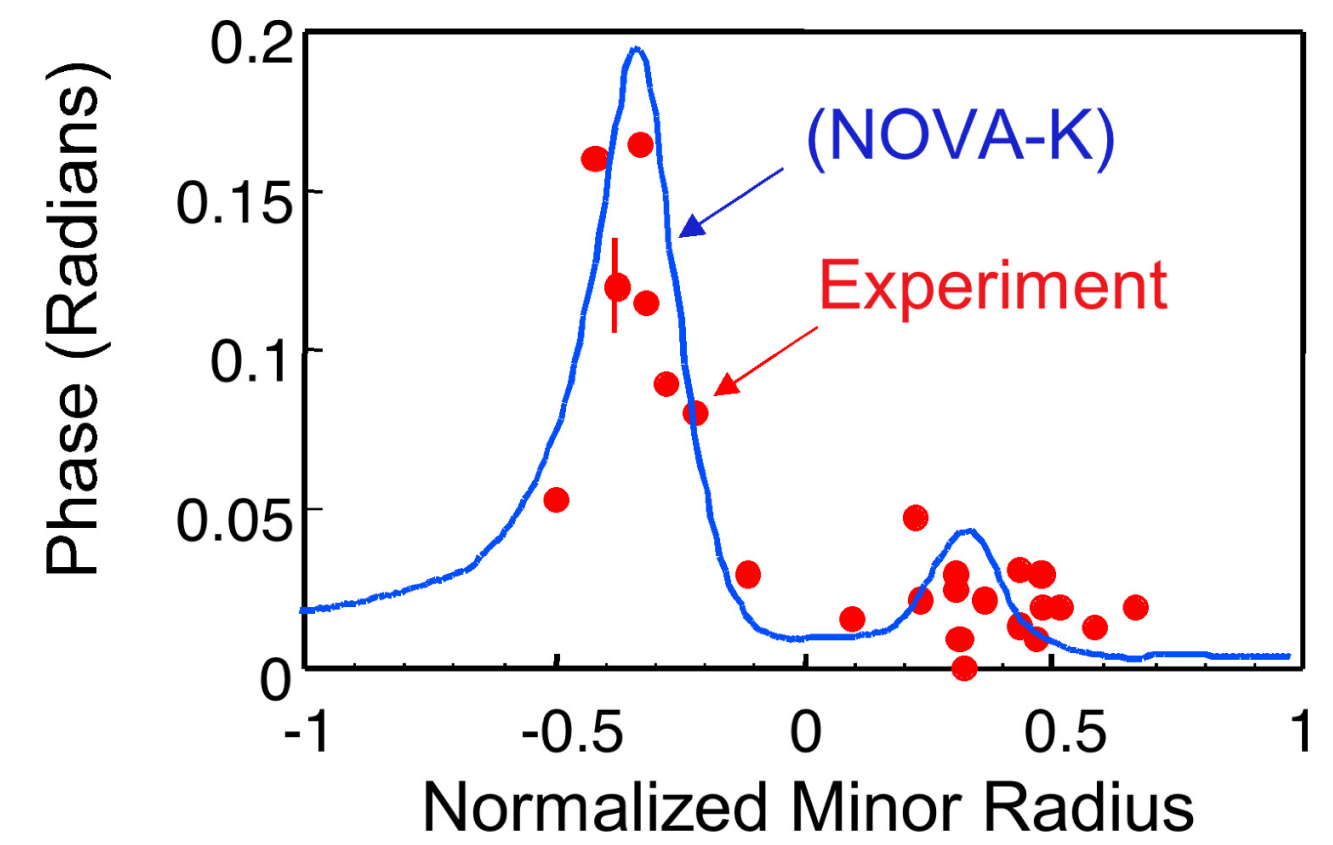

(a)

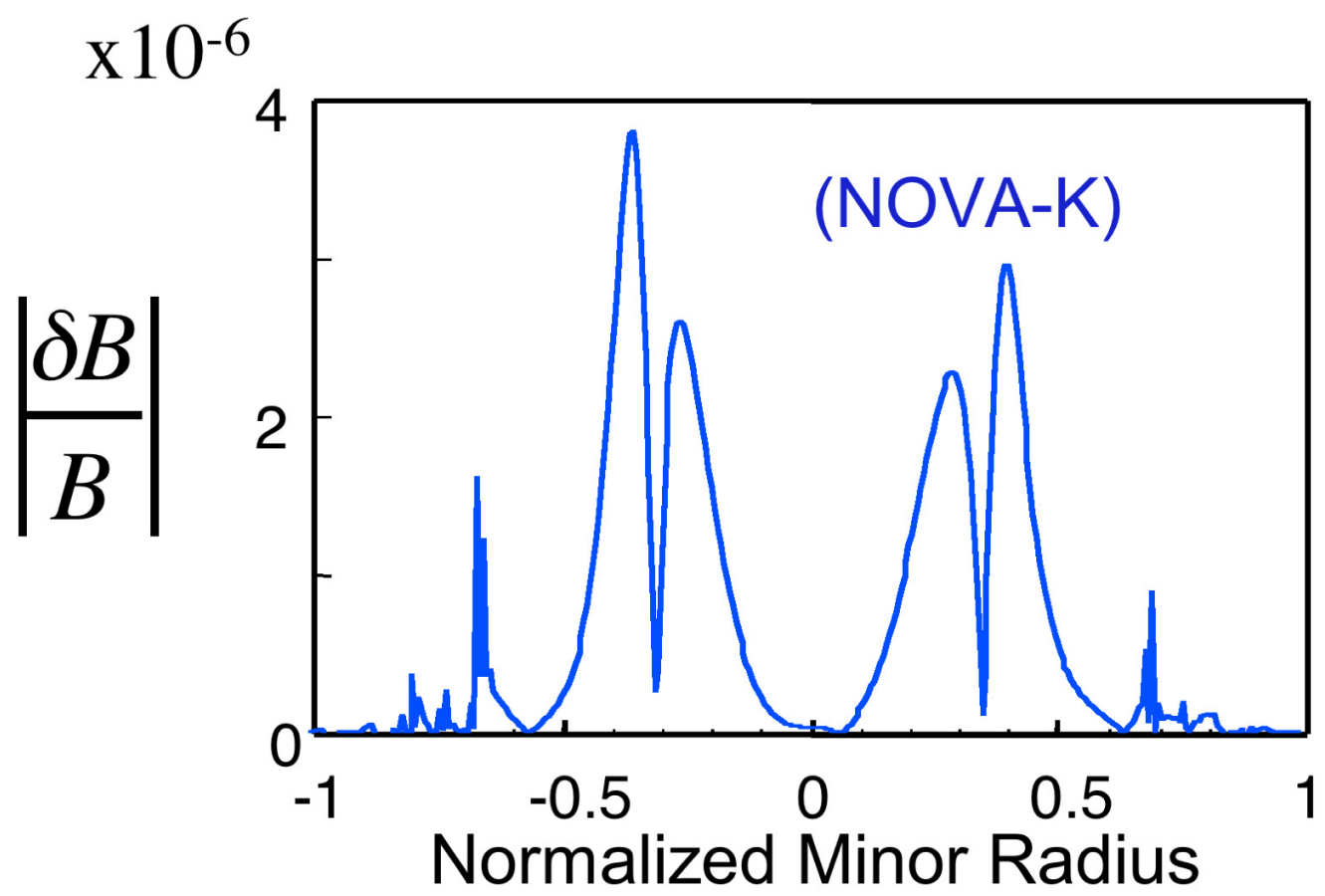

(b)

Fig. 5.6 Measured radial structure of density fluctuations (a) for the $n=2$ mode compared to the theoretically expected radial mode structure for the $n=2$ Cascade mode according to the NOVA-K code. In (b) the magnetic fluctuation level from NOVA-K is also shown, indicating the cylindrical nature of the mode from the near equal amplitude on the high and low field side of the magnetic axis. 


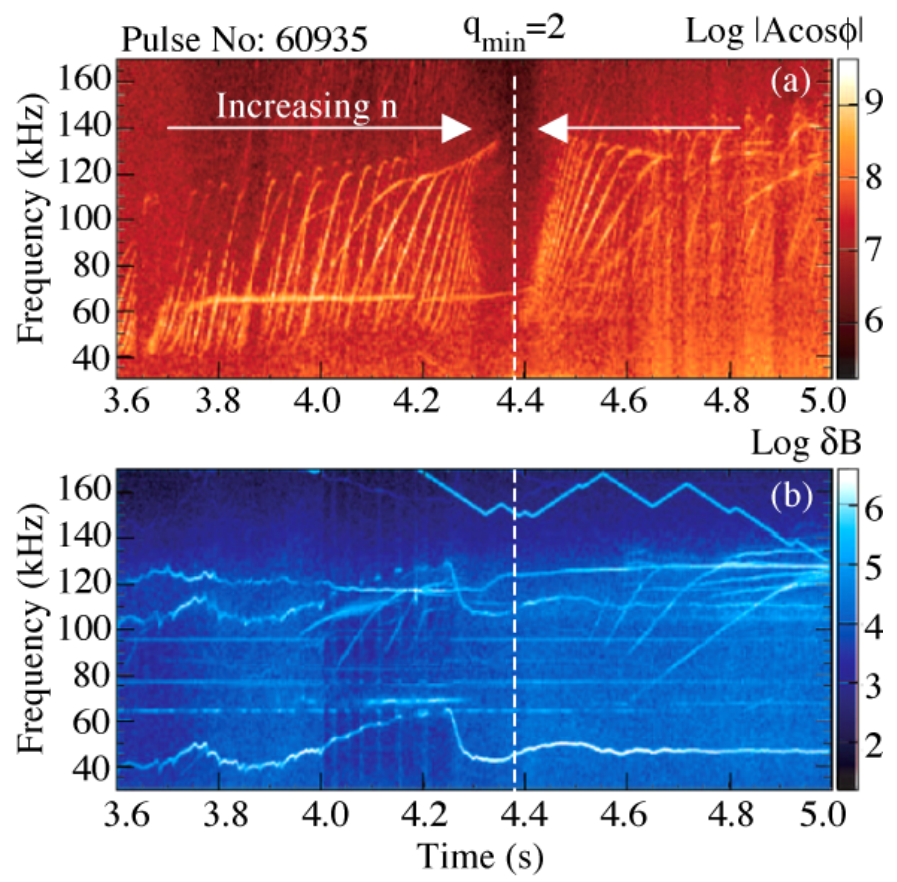

Fig. 5.7 Amplitude of plasma perturbations as function of time and frequency measured in JET discharge. (top) Interferometer measurements with microwave beam of $45.21 \mathrm{GHz}$ along plasma mid-plane showing up to $\mathrm{n}=16$ modes and (bottom) measurements with external magnetic pick-up coils with $n=3-5$. 

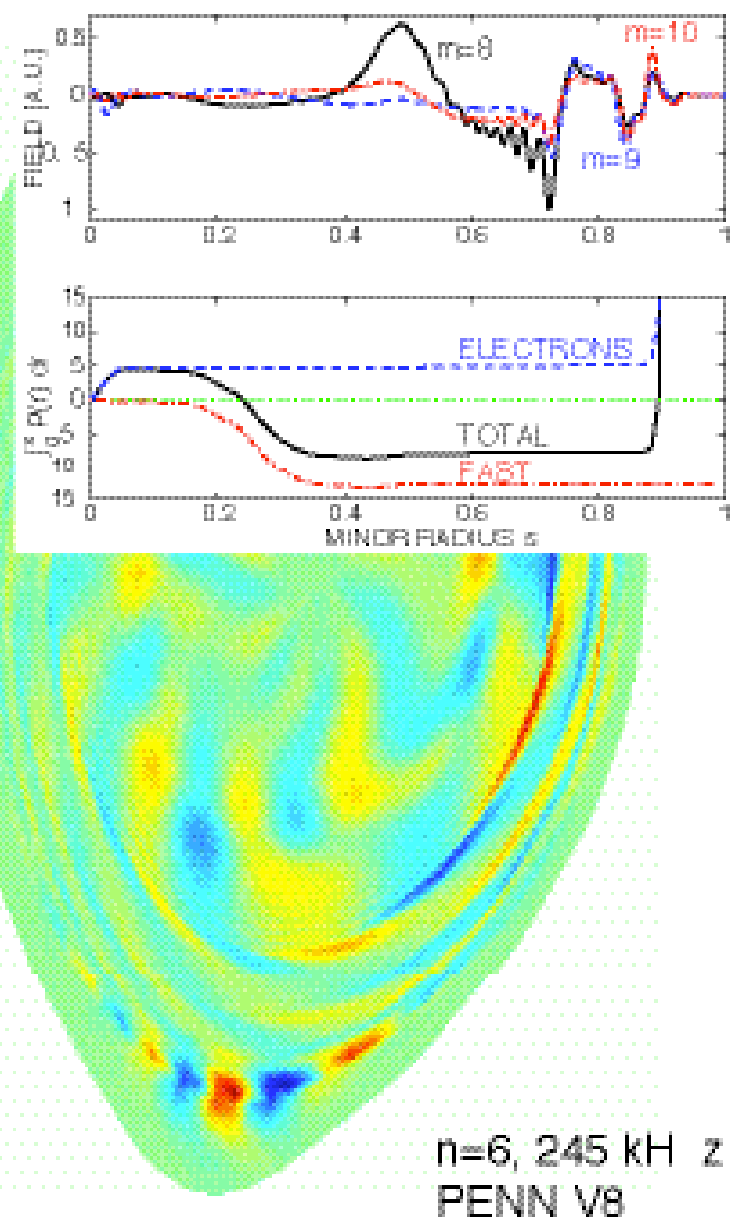

Fig. 5.8 Wave field of a kinetic Alfvén Eigenmode (KAE) with intermediate mode number $\mathrm{n}=6$ in JET. The power transfers show that this radially extended mode is driven by the fast ions in the interval $s=0.2-0.3$ and is stabilized by the electron Landau damping of kinetic Alfvén waves in the core $s<0.05$ and the edge $s \cong 0.9$. Radiative damping and drive in $s=0.55$ 0.75 are small in comparison. 
AE damping vs plasma shaping
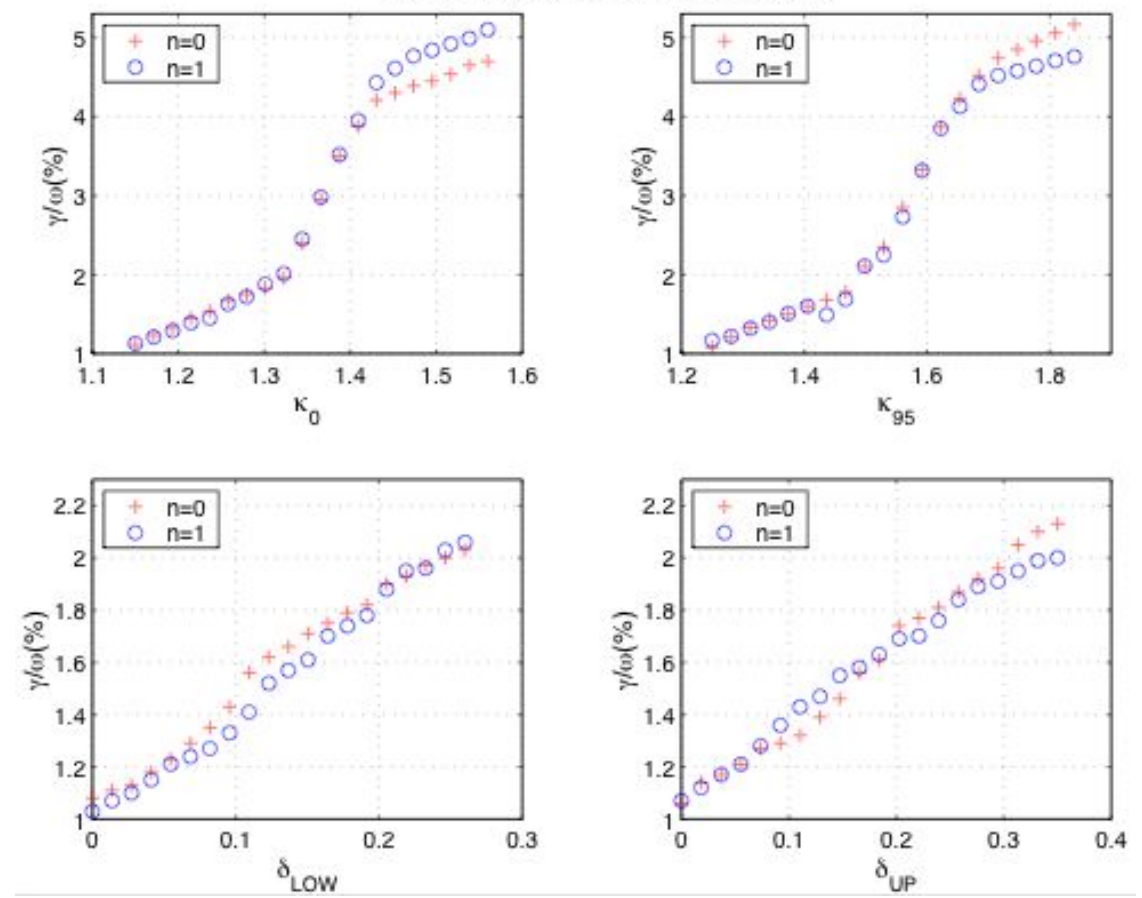

Fig. 5.9 Variation of the AE damping rate measured as a function of the elongation $\kappa$ and the triangularity $\delta$ of limiter JET pulses, for similar ohmic plasmas with $\mathrm{q}_{95} \cong 4$ and $\mathrm{q}_{0} \cong 0.9$. We notice a sharp variation in $\gamma / \omega$ as a function of the elongation around $\kappa_{95} \cong 1.5$ and $\kappa_{0} \cong 1.35$, whereas $\gamma / \omega$ varies approximately linearly with the triangularity. 


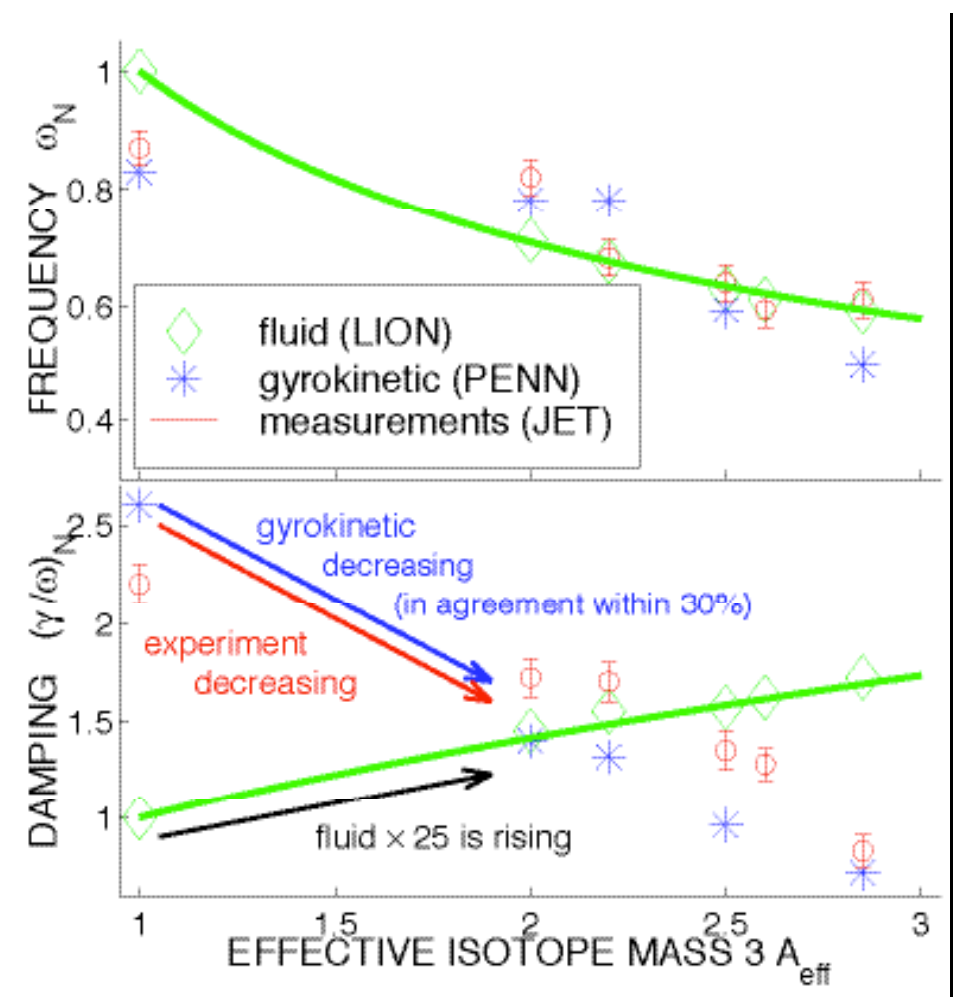

Fig. 5.10 Measured and computed effect of the ion mass on the frequency (top) and the damping rate (botton) of $n=1$ TAE modes in similar JET limiter plasmas. Note that the damping rate from fluid models has been multiplied by 25 to compare the scaling. 


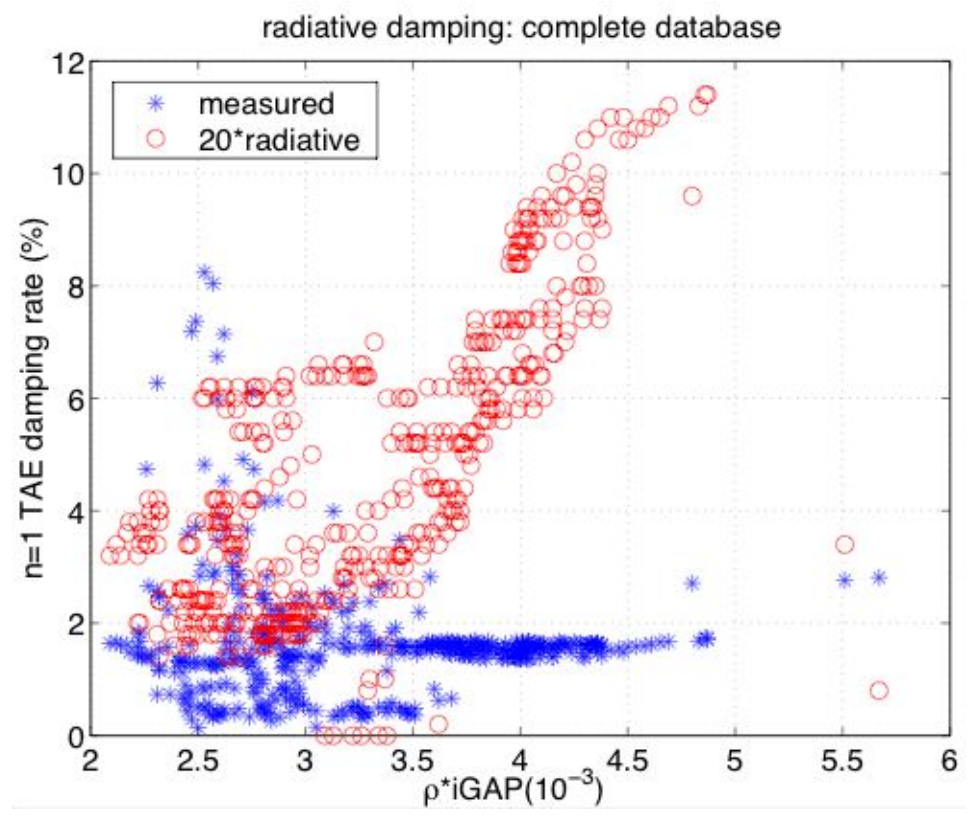

Fig. 5.11 Damping rate for a $n=1$ TAE measured as a function of the normalized Larmor radius at the gap location $(\rho / a)_{\text {gap }}$, using an ohmic limiter plasma in JET for a comparison with the radiative damping model multiplied by 20 to compare the scaling. 

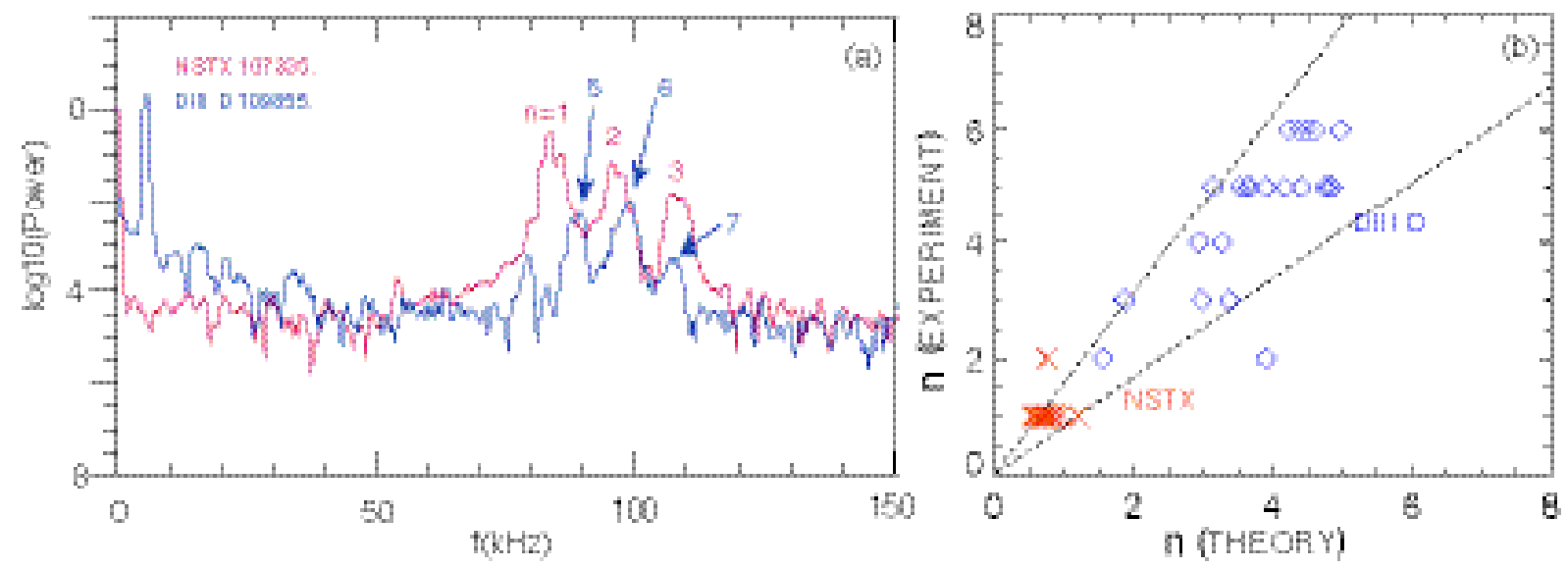

Fig. 5.12 The comparison of the Mirnov signal spectrum in NSTX and DIII-D similarity experiments (a) shows higher observed mode numbers of unstable TAEs in DIII-D. The most unstable toroidal mode numbers experimentally observed are here tested against the scaling $\mathrm{n} \sim \mathrm{a} / \mathrm{q}^{2}(\mathrm{~b})$. 


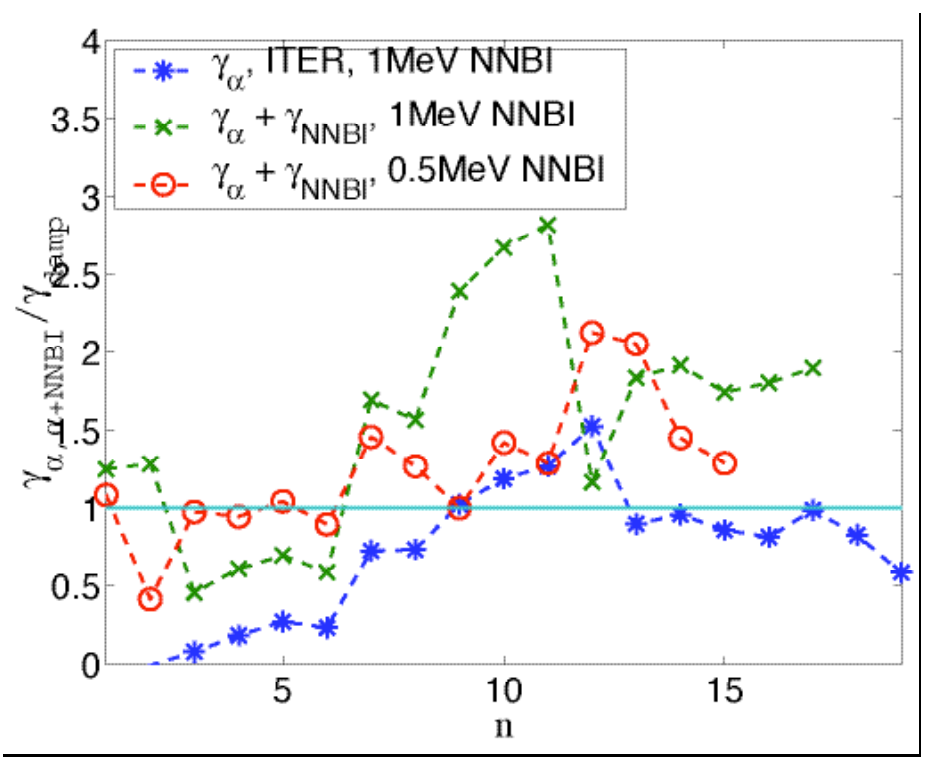

Fig. 5.13 NOVA-K predictions of TAE growth rates for ITER. Shown on the left are the maximum ratio of drive to damping for the cases where there is drive from alpha particles alone and when the drive is from both alpha-particles and $1 \mathrm{MeV}$ neutral beam ions. The dependences are plotted as functions of the mode toroidal number $\mathrm{n}$. 

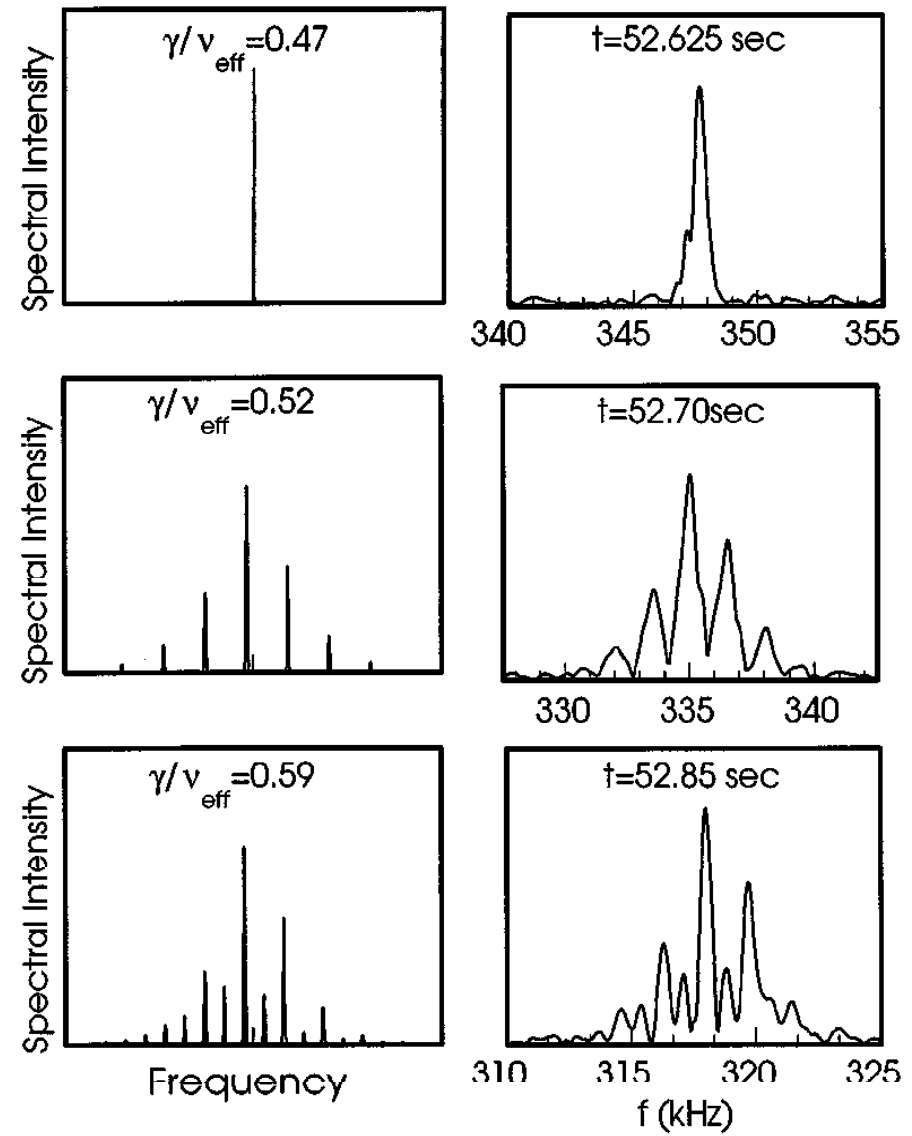

Fig. 5.14 Nonlinear evolution and pitchfork splitting of an ICRH driven $n=7$ TAE in JET [187]. The spectral line and the period doubling bifurcation are followed in snapshots of the mode power spectrum. Left: calculated power spectrum of the saturated solution of Eq. (1) in Ref. [187], as the parameter $\gamma / v_{\text {eff }}$ increases. Right: time evolution of experimental spectrum of magnetic activity during the increase of the ICRH power (JET shot No. 40328). 


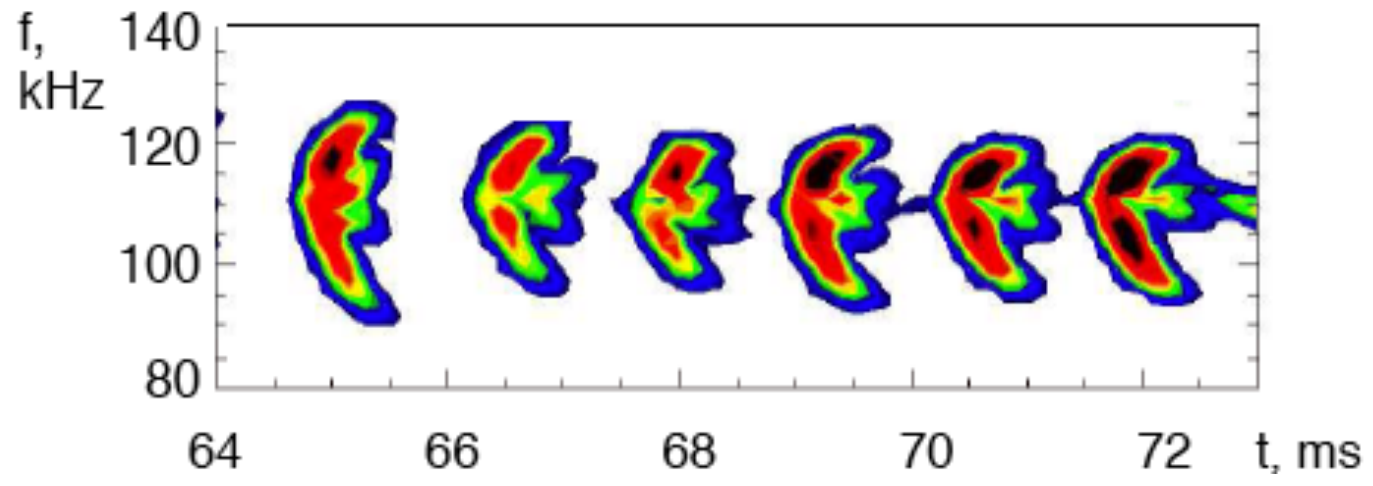

Fig. 5.15 Magnetic spectrogram showing frequency sweeping $n=1$ core-localized mode in MAST \# 5568. 

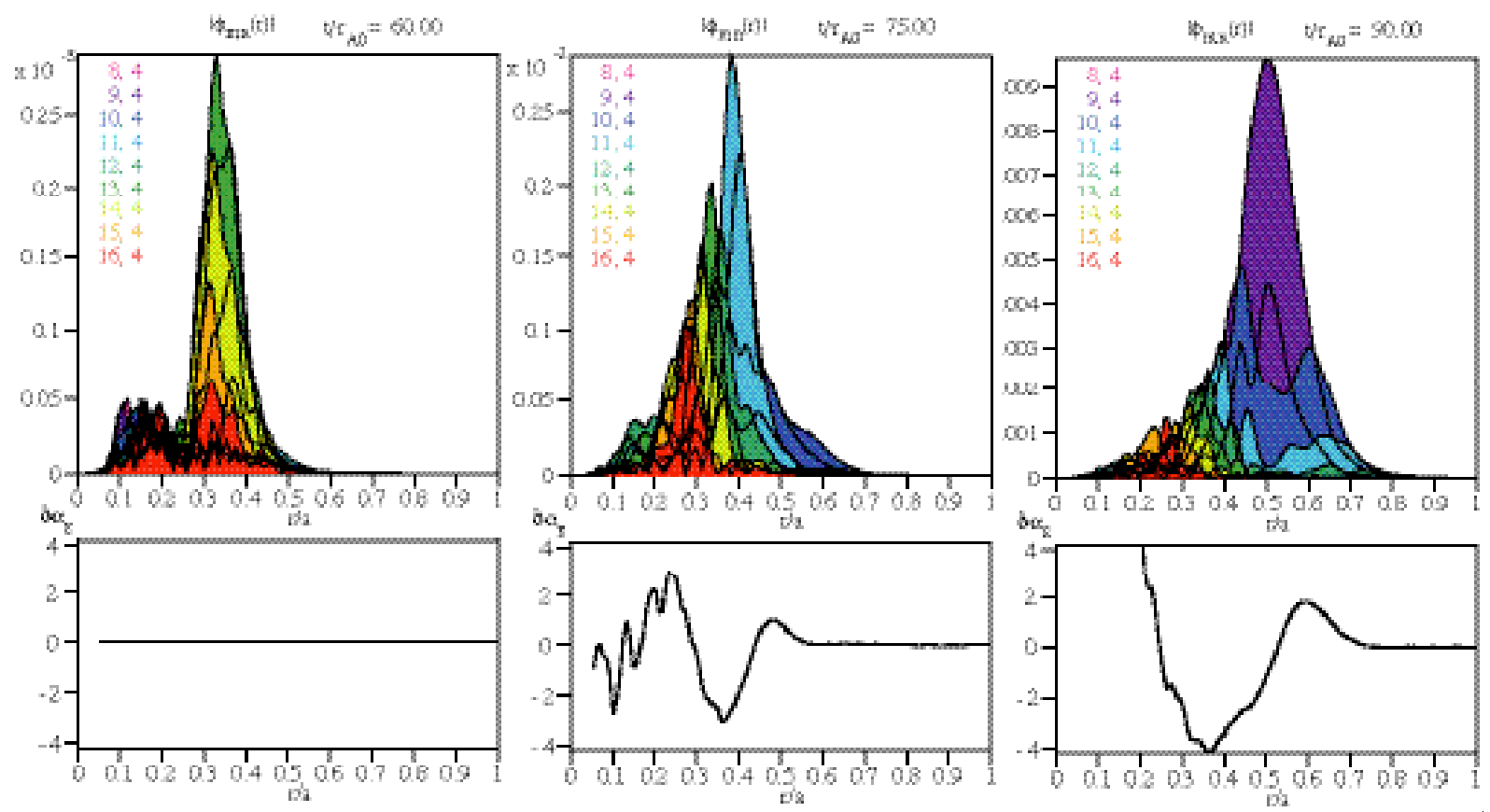

Fig. 5.16 Time evolution of the EPM radial structure, decomposed in poloidal Fourier harmonics. Here $\tau_{\mathrm{A} 0}=\mathrm{R}_{0} / \mathrm{v}_{\mathrm{A}}(\mathrm{r}=0)$. The toroidal mode number is $\mathrm{n}=4$. The nonlinear modification of $\alpha_{E}=-R_{0} q^{2}\left(d \beta_{E} / d r\right)$ is also shown. 